\title{
Impulsive noise modeling and robust receiver design
}

\author{
Laurent Clavier ${ }^{1 *} \mathbb{D}$, Gareth W. Peters ${ }^{2}$, François Septier ${ }^{3}$ and Ido Nevat ${ }^{4}$
}

\author{
*Correspondence: \\ laurent. \\ clavier@imt-lille-douai.fr \\ ${ }^{1}$ IMT Lille Douai, Univ. Lille, \\ CNRS, UMR 8520 - IEMN, \\ 59000 Lille, France \\ Full list of author information \\ is available at the end of the \\ article
}

\begin{abstract}
Interference is an important limitation in many communication systems. It has been shown in many situations that the popular Gaussian approximation is not adequate and interference exhibits an impulsive behavior. This paper surveys the different statistical models proposed for such an interference, that can generally be unified using the class of sub-exponential family of distributions, and its impact on the receiver design. Visualizing the optimal decision boundaries allows one to show the non linear effect induced by impulsive noise models, which explains the significant loss in receiver performance designed under the standard Gaussian approximation. This motivates the need to develop new receivers. We propose a framework to design receivers robust to a variety of interference types, both Gaussian and non-Gaussian. We explore three ways of thinking about such receiver designs: a linear approach; by approximating the noise plus interference distribution; and by mimicking the decision rule distribution directly. Except for the linear approach, the other designs are capable of replicating the nontrivial optimal decision regions to different extents. The new detection algorithms are evaluated via Monte Carlo simulations. We focus on four efficient architectures, including the parameter estimations: Myriad, Normal Inverse Gaussian, $p$-norm and a direct estimation of the likelihood ratio function. They exhibit good performance, close to the optimal, in a large range of situations demonstrating they may be considered as robust decision rules in the presence of heavy tailed or impulsive interference environments.
\end{abstract}

Keywords: Interference, Impulsiveness, Robust receiver, Normal Inverse Gaussian distributions

\section{Introduction}

Wireless communication systems are usually designed assuming Gaussian noise. This fundamentally impacts many solutions that are used in transmitters and receivers. This is especially the case when it comes to the design of the receiver decision strategy which is typically directly derived from Gaussian assumptions. When these assumptions are not satisfied, or the interference is far from Gaussian, the receiver is significantly suboptimal in its performance. One frequently encountered type of noise is designated by the term impulsive. However, this term covers a lot of different statistical models and each model gives rise to many different communication strategies. In this paper, we aim to give insight in the choices that can be made to model the noise and design an adapted receiver. We want this receiver to be flexible enough in order to exhibit performance author(s) and the source, provide a link to the Creative Commons licence, and indicate if changes were made. The images or other third party material in this article are included in the article's Creative Commons licence, unless indicated otherwise in a credit line to the material. If material is not included in the article's Creative Commons licence and your intended use is not permitted by statutory regulation or exceeds the permitted use, you will need to obtain permission directly from the copyright holder. To view a copy of this licence, visit http:// creativecommons.org/licenses/by/4.0/. 
close to the optimal in many situations, from purely Gaussian noise to highly impulsive situations.

Impulsive noises have an important impact in many communication settings, as can be seen in a very recent literature: power-line communications $[1,2]$ or digital subscriber loop [3]; wireless networks and OFDM [4, 5]; wireless sensor networks [6-8]; acoustic communication $[9,10]$; it is also frequently encountered in different vehicular communications scenarios [11].

In the literature, a variety of statistical models have been proposed for the interference, but it remains challenging to unify these frameworks and to select between the different choices for a given application. One commonly encountered feature is that the noise can exhibit an impulsive behaviour. This means that large noise values can appear from time to time $[12,13]$. These large samples have a dramatic impact on the receiver which is not supposed to see such events and fails to recover the proper data.

In this paper, we first make a survey of interference statistical models and receiver designs that have been developed in the wireless communications literature since the works from Middleton in 1977. We then propose to characterize impulsiveness in a mathematical way according to an overarching family of models: the subexponential family. This provides valuable insight into general properties of impulsive interference, which were previously proposed in seemingly unrelated studies.

We then consider the detection problem in a block fading scenario. Each data symbol is transmitted over wireless channels and $K$ versions of each symbol are received. This transmission structure can be motivated by many different practical wireless communication systems, for example transmission for a rake receiver [14], a single-input-multiple-output system [15], in a cooperative communication system involving multiple relays [16] or in impulse radio Ultra Wide Band systems where repetitions of the transmitted symbol occur [17]. However the design rule that we propose can also be applied when channel coding $[18,19]$ or CDMA are implied which induce higher dimension problems. However, focusing on a simple scheme allows us to better understand the underlying phenomena in detail.

The main objective of this paper is not to introduce new receiver designs but to explore the state of the art of receiver design from a wide variety of literatures in great detail with regard to their decision regions and performance, adaptibility, robustness and flexibility under varying impulsive environments. We attempt to explain behaviours of classes of existing receivers so that one can better understand their properties relative to each other and make appropriate choices in a specific context. The main contributions can be summarized as:

1 We first present a review of existing works about impulsive interference modelling and receiver design. We define three ways to design the receiver: linear approach, approximation of the interference PDF or approximation of the log-likelihood ratio (LLR).

2 We propose in Sect. 3.3 a definition for impulsive interference using the sub-exponential family. It is important because impulsiveness in communication is widely used but rarely defined. We also propose in Sect. 3.4 to visualize the effect of impulsive noise on the decision regions of an optimal receiver. 
3 We propose in Sect. 4 design strategies for robust receivers. Some are already known (Myriad filter [20, 21], $p$-norm [22]) but we are interested by their efficiency, accuracy and robustness when the noise characteristics change. We introduce estimation algorithms to ensure their adaptation capabilities. We also extend the solution we introduced in [23] based on the Normal Inverse Gaussian (NIG) family and propose a receiver that directly estimates the log-likelihood ratio function [19, 24, 25].

4. We finally evaluate through simulations the robustness of several receivers when the interference impulsiveness varies or when the noise model is changed in the case of linear, myriad, $p$-norm, NIG and LLR-based receivers. We also study the impact of the length of the training sequence.

Since we do not assume that we know a priori the interference distribution or because general models result in complicated (or non existing) analytical expressions for the $\mathrm{PDF}$, it is not possible to have a fully analytical performance study. We consequently rely on Monte Carlo simulations to assess the performance. However, in the analysis we provide we do consider a large spectrum of interference settings so that our results are general and our findings wide ranging in their coverage. Besides our theoretical interference analysis makes clear the reasons why a receiver design works and when it will fail.

\section{Method and organisation of the paper}

The work is organised in three main steps:

- an important effort was made to understand previous works on interference and receiver and especially to understand the impact of non Gaussian noises on the receiver design. First we did an extensive study of the state of the art on the interference models, presented in Sect. 3.2. This allowed us to identify properties of impulsive interference (sub-exponential family, Sect. 3.3). We then proposed a clear illustration of the effect of non Gaussian noise on the optimal decision that the receiver should make (Sect. 3.4.1). Along with a review on receiver design (Sect. 3.4.2), we then proposed a classification of receivers (linear, interference PDF based or LLR based);

- we then proposed relevant receiver strategies (Myriad filter, $p$-norm, Normal Inverse Gaussian and LLR approximation) and the necessary parameter estimations (Sect. 4). If we assumed that the channel is known (perfect channel state information) at the receiver, on the contrary, no information about the noise is known a priori;

- the different approaches are finally evaluated through simulations under different interference scenarios and compared to a linear approach and to the optimal receiver (Sect. 5). We showed that the proposed receivers can adapt to a wide range of interference settings, but the myriad filter that do not adapt well to a decrease in impulsiveness.

In the rest of the paper we use the following notation: a bold letter (for instance $\mathbf{Y}, \mathbf{h}$ ) refers to a vector ; if not bold (for instance $s, Y, h$ ), it refers to a scalar. A random vector is an uppercase bold letter $(\mathbf{Y})$ when a lowercase bold letter is a specific realization $(\mathbf{y})$. $\mathbb{P}_{\mathbf{Y}}(\mathbf{y} \mid s ; \mathbf{h})$ refers to the probability of the event $\mathbf{Y}=\mathbf{y}$ knowing $s$ and $\mathbf{h}$. For a continuous 
random variable it will be the probability density function denoted as $f_{\mathbf{Y}}(\mathbf{y})$. $\hat{s}$ is the estimated value of $s$.

$\mathbf{Y}$ refers to the received vector, $\mathbf{h}$ to the channel realization, $K$ is the number of repetitions of the transmitted bit, $s$ is the transmitted information bit, $\mathbf{I}$ is the interference vector ans $\mathbf{N}$ is the thermal noise vector. Each of the previously mentioned vector is of dimension $K \times 1$.

\section{System model, interference modeling and receiver review}

\subsection{System model}

For a single transmitted symbol, the received signal $\mathbf{Y} \in \mathbb{R}^{K}$ is:

$$
\mathbf{Y}=s \mathbf{h}+\mathbf{I}+\mathbf{N},
$$

where $s$ is the unknown transmitted symbol, $\mathbf{h} \in \mathbb{R}^{K}$ is the block fading channel coefficients, $\mathbf{I} \in \mathbb{R}^{K}$ is the interference and $\mathbf{N} \in \mathbb{R}^{K}$ is the thermal noise with its elements $N_{k} \stackrel{\text { i.i.d. }}{\sim} \mathcal{N}\left(0, \sigma^{2}\right)$.

The optimal receiver in terms of minimizing the Bit Error Rate (BER) is the Maximum Likelihood (ML) detector. It is given by the solution to the following optimization problem:

$$
\widehat{s}=\underset{s \in \Omega}{\arg \max } \mathbb{P}_{\mathbf{Y}}(\mathbf{y} \mid s ; \mathbf{h})=\underset{s \in \Omega}{\arg \max } \sum_{k=1}^{K} \log \mathbb{P}_{Y_{k}}\left(y \mid s ; h_{k}\right),
$$

the second equality assuming independent noise samples.

Calculating (2) requires the evaluation of the measure $\mathbb{P}_{\mathbf{Y}}(\mathbf{y} \mid s ; \mathbf{h})$. In a general approach, this involves several steps: (a) specify the representation of the impulsive noise I, either by its characteristic function (CF) or distribution function (when it exists in closed form); ( $b$ ) if $\mathbf{I}$ is known only through its CF (like for $\alpha$-stable distributions), find its Probability Density Function (PDF) through the Fourier transform; (c) calculate the PDF of the total interference $I_{k}+N_{k}$, via a convolution:

$$
f_{I_{k}+N_{k}}(\zeta)=\frac{1}{2 \pi} \int_{-\infty}^{\infty} f_{N}(\tau) \int_{-\infty}^{\infty} \varphi_{I_{k}}(t) e^{-i(\zeta-\tau) t} \mathrm{~d} t \mathrm{~d} \tau, \quad \forall k \in\{1, \ldots, K\} ;
$$

(d) conditional on the channel state information, find the likelihood function as a function of $s$ denoted by $\mathbb{P}_{\mathbf{Y}}(\mathbf{y} \mid s ; \mathbf{h})$ and choose $s$ that maximizes the likelihood.

The description of these different steps serves to highlight the difficulties one can encounter when designing a receiver. Firstly, specifying the PDF of the interference can simply be a priori impossible because the transmission environment is not predictable. Secondly complexity can be an important issue when deriving the likelihood, for example with Middleton or $\alpha$-stable PDF. This challenges how one approaches receiver design.

We consider the binary case only and make the following assumptions:

1 The unknown transmitted symbol, $s$, is defined on a discrete support $\Omega=\{-1,1\}$ with equally likely elements to be transmitted.

2 The block fading channel coefficients define a random vector (RV) denoted by $\mathbf{h} \in \mathbb{R}^{K}$. The distribution of the coefficients depends on the considered channel 
Table 1 Different interference models

\begin{tabular}{|c|c|c|}
\hline $\begin{array}{l}\text { Where does the model } \\
\text { come from? }\end{array}$ & What are the main results? & $\begin{array}{l}\text { Examples, comments, } \\
\text { simplification }\end{array}$ \\
\hline Work from Middleton $[32,33]$ & $\begin{array}{l}\text { Distribution expressed as infinite } \\
\text { series }\end{array}$ & $\begin{array}{l}\text { Simplification to the most significant } \\
\text { terms: } \epsilon \text {-contaminated }[115,116] \text {, } \\
\text { Bernoulli-Gaussian }[39,40,117, \\
\text { 118], Gaussian mixtures }[37,54], \\
\text { Markov }[41,42]\end{array}$ \\
\hline $\begin{array}{l}\text { Empirical approach based on fitting } \\
\text { of data and improvement of the } \\
\text { receiver }\end{array}$ & Many different distributions & $\begin{array}{l}\text { Laplace [50], Cauchy [17], Cauchy- } \\
\text { Gaussian mixture [55], Generalized } \\
\text { Gaussian [51-53], Normal Inverse } \\
\text { Gaussian [23] }\end{array}$ \\
\hline $\begin{array}{l}\text { Based on stochastic geometry } \\
{[62-64,119]}\end{array}$ & $\begin{array}{l}\text { Distribution expressed as infinite } \\
\text { series }\end{array}$ & $\begin{array}{l}\text { If no near-field effect, falls in the } \\
\text { attraction domain of an } \alpha \text {-stable } \\
\text { distribution }[17,65,74]\end{array}$ \\
\hline
\end{tabular}

model (e.g. Rayleigh, Nakagami, Rician etc.). We assume perfect channel state information at the receiver.

3 The impulsive interference is denoted by a RV $\mathbf{I} \in \mathbb{R}^{K}$ in which all elements are assumed independent and identically distributed (i.i.d.). This assumption is verified in some settings [26] but is an open discussion and can depend on the scenario considered [27, 28]. The physical layer and signal processing, like discussed in [29] for the passband-to-baseband conversion, can lead to different dependence structure. Treating the dependent case, however, remains out of the scope of this paper.

4. The thermal noise at the receiver is a $\mathrm{RV} \mathbf{N} \in \mathbb{R}^{K}$ in which all elements are assumed i.i.d. with a Gaussian distribution, $N_{k} \stackrel{\text { i.i.d. }}{\sim} \mathcal{N}\left(0, \sigma^{2}\right)$.

5 The interference is independent of the thermal noise, i.e., $\mathbf{I} \perp \mathbf{N}$.

With these assumptions, the ML detector in (2) is given by:

$$
\sum_{k=1}^{K} \log \frac{\mathbb{P}_{Y_{k}}\left(y \mid s=1, h_{k}\right)}{\mathbb{P}_{Y_{k}}\left(y \mid s=-1, h_{k}\right)} \underset{\widehat{s}=-1}{\stackrel{\widehat{s}=1}{\gtrless} 0 .}
$$

\subsection{Standard interference modeling}

In many previous papers, it has been shown that the interference term is not adequately modelled with a simple Gaussian distribution assumption. We present here some of the key results in this regard that we summarize in Table 1.

\subsubsection{Middleton model}

We can trace back some works on non Gaussian noise to 1960 [30] and 1972 [31] about atmospheric noise. Assuming Poisson distributed sources, the CF of the impulsive noise can be obtained. Furthermore, appropriate assumptions on the transmission medium and source waveforms allow one to obtain the interference PDF. A similar approach based on the CF was used by Middleton [32,33] who obtained more general expressions based on series expansions. He classified interference in two main categories depending if the noise bandwidth is less than the useful signal (class A) or greater (class B). Class C is a sum of class A and B. 
Middleton models have been widely used in different contexts [34, 35]. It is clear however that this popular model is challenging to work with in receiver design since the density function involves infinite sums. Furthermore, for the class B, the alternating sign of the series of summands make truncation challenging to ensure the truncated representation is still a positive quantity for probability calculations. Besides, the individual summand terms can become for large indexes challenging computationally to evaluate, hence not easily computable online in real receiver settings. Consequently, several approximation models have been proposed. The main approach is to consider only the most significant terms. For instance, it is claimed in [36] that, in many situations for the class A, two or three terms can be sufficient to obtain a good approximation leading to a Gaussian mixture [37]. Moreover, in a different context but relevant for how interference characterization in Sect. 3.3, it is mentioned in [38] that a Gaussian mixture can capture multi-modality, asymmetry, heavy tails, which makes it an appealing solution. The two terms case is often denoted as the $\epsilon$-contaminated noise, see [35]. In this case the interference PDF is $\mathbb{P}(x)=(1-p) \mathcal{N}\left(0, \sigma^{2}\right)+p \mathcal{N}\left(0, \kappa \sigma^{2}\right)$, where $p$ denotes the probability to have an impulse, distributed from a Normal with variance $\kappa \sigma^{2}$ while $(1-p)$ gives the probability to only have the Gaussian noise with variance $\sigma^{2}$. Usually, $p$ is small $(p=0.01)$ and $\kappa$ large $(\kappa=50,100)$. The $\epsilon$-contaminated model can also be expressed in the form of a Bernoulli-Gaussian noise [39, 40]. Noise plus interference is expressed as $n+b i$, where $n$ is the Gaussian noise, always present, and $b$ a Bernoulli random variable with parameter $p=\mathbb{P}(b=1)$ representing the frequency of impulsive noise $i$ occurrence. Usually, $i$ is represented by a Gaussian random variable with a larger variance than $n$.

In [41-43], the class A model is represented by a Markov process: the noise distribution depends on the state of the process. It reduces to the $\epsilon$-contaminated case when only two states are present, but with an additional feature of time dependence structure, see [44].

The popular Class B model can be approximated by an $\alpha$-stable distribution [33], still difficult to use in practice but that we will introduce in Sect. 3.2.3.

\subsubsection{Empirical approaches}

More recently, many works have been done concerning Time Hopping Ultra Wide Band (TH-UWB) [45]. After showing that the standard Gaussian model is not accurate [46], non Gaussian models were developed. To specify the representation of the impulsive noise I for TH-UWB, Forouzan et al. [47] have studied the perfect power control case, synchronized or unsynchronized, and derived a tractable expression for the total interference PDF. To do so, they approximated the interference generated by one user. Sabattini et al. [48] have considered the CF. These works do not solve the complexity issues and remain very specific to the studied cases. Durisi and Benedetto [49] have simplified the analysis, only considering the moments to derive the error probability. However, this does not allow an efficient receiver design. Many works have also proposed empirical choices that allow analytical analysis of the receiver, justified by simulations, observations of the estimated PDF and/or gains in BER. The main solutions that have been proposed include Gaussian-Laplace mixture [50], Generalized Gaussian [51-53], Gaussian mixtures [54] or Cauchy-Gaussian mixture [55]. In this last paper it is mentioned that 
the heavier tail of the Gaussian Mixture allows better performance than the Laplace approach. Some surveys can be found in $[56,57]$.

All these approaches target a specific class of interference and are not supposed to be robust or adaptive to changing interference environments. Another class of model of direct relevance to interference modelling is the $\alpha$-stable. It has often been used in the UWB context $[17,56,58-61]$. But on the contrary to the previously discussed approaches, it relies (when no power control is done) on a theoretical derivation (that can be related to a physical interpretation), closely linked to the Middleton's work and finding its foundation in stochastic geometry [62-64].

\subsubsection{Stochastic geometry and $\alpha$-stable}

Although the first papers were published in the nineties [65-67], the analysis of networks has recently attracted a lot of works relying on stochastic geometry. As in Middleton's work, interferers are assumed spatially distributed according to a Poisson field. In this context, the distribution of interference is expressed as

$$
I=\sum_{i \in \Omega} l\left(d_{i}\right) \cdot \mathcal{Q}_{i}
$$

where $d_{i}$ is the distance between interferer $i$ and the destination and $l(d)$ the attenuation as a function of the distance; a classical model is $l_{\gamma, \epsilon}(d)=d^{-\gamma} 1_{\mathrm{r} \geq \epsilon}, \mathrm{d} \in \mathbb{R}^{+}$where $\gamma$ is the channel attenuation coefficient; $\epsilon$ accounts for a minimum distance between the receiver and the transmitter for physical reasons or due to some MAC layer protocol like carrier sensing; $Q_{i}$ accommodates various propagation effects such as multipath fading and shadowing as well as the physical layer of the transmitters and the receiver; and $\Omega$ is the set of interferers.

If applied in an ad hoc network, an unbounded received power assumption makes the interference fall in the attraction domain of a stable law. This unbounded assumption means taking the limit as $\epsilon \rightarrow 0$; in that case the received power tends to infinity when $d$ tends towards zero. The accuracy of the approximation has been questioned in $[68,69]$, but working without the unbounded received power assumption does not allow an analytical derivation of the characteristic function [64, 70]. A truncated $\alpha$-stable distribution is proposed in $[13,71]$ to solve the infinite variance problem.

This result can be seen as a consequence of the generalized central limit theorem [72, 73]. The main advantage of the heavy tailed stable distributions is their ability to represent rare events. In many communication situations, these events are in fact those that will limit the system performance. The traditional Gaussian distribution ignores them leading to poor results.

The proof of this result is generally done considering the log-CF of the total interference, see for instance $[17,65,74]$, which can be written as:

$$
\psi_{I}(\omega)=\log \left(\mathbb{E}\left[e^{j \omega^{T} I}\right]\right)=-\delta^{\alpha}|\omega|^{\alpha},
$$

where $I$ is the total interference and $T$ denotes the transpose. The right term is the logcharacteristic function of a symmetric $\alpha$-stable $(\mathrm{S} \alpha \mathrm{S})$ random variable with dispersion $\delta$. Another solution for the proof, based on the Lepage series, was proposed in [67]. 
This area of research is still active. Problems concerning the non homogeneous position of users are studied, for instance based on cluster point process $[75,76]$ for general ad hoc networks or Poisson hole process for cognitive radio [77]. The dependence structure of interference is also attracting many works [78-80]: it is an important feature for the network analysis but difficult to handle. Mahmood et al. studied the dependence structure at the baseband between two $\alpha$-stable interference sample $[29,81]$ and showed that with proper sampling they could be made independent.

If the proposed framework offers an efficient tool for the network performance evaluation, a simple (with a tractable expression) and accurate model is still to be found [82]. It is probably unrealistic anyway to believe that one simple model will be able to cover all the possible situations. Therefore if we aim to design a robust receiver, we need it to adapt to the context and the changing interference environments that may be faced over space or time.

\subsection{Impusive interference modelling under the unified family of the sub-exponential models}

The previous discussions on background literature have detailed specific examples of impulsive interference models. Unifying these different concepts in a general class can help communication strategies, which are flexible and can adapt to a wide range of interference environments. In this section we propose a general class of mathematical models for impulsive interference which encompasses examples discussed previously: the sub-exponential family, extended to the entire real line (not only $\mathbb{R}^{+}$). In [12], interference distributions are classified in superexponentially, exponentially or subexponentially decaying tails to explain the reason why a large deviation can appear in the total interference: due to many small contributions or a single large one. Similarly, Weber et al. [83] justify the tightness of a lower bound on the transmission capacity in an ad hoc network, using the fact that a sum of iid random variables typically achieve large values due to one or more large summands rather than a large number of moderate value summands; such observations are aligned naturally with the property of distributions in the sub-exponential class of interference models.

A way to characterize impulsive interference is via considering probability distributions whose tails are not exponentially bounded: that is, they have heavier tails than the exponential distribution. Indeed, in impulsive interference we can observe large values that rarely appear. Such a behavior can be accurately represented with a so-called heavy tailed distribution. It is useful to relate this class of distributions to the behaviour of the moment generating function (MGF) as one often considers heavy tailed models as those with non-finite mean or variance or higher order moments. In doing so, we see that an interference distribution which is heavy-tailed under this characterization will have a MGF that is finite in some right neighborhood of the origin if and only if the following bound on the complementary cumulative distribution function $\bar{F}(x)=\mathbb{P}(X>x)$ holds for some positive real numbers $M$ and $t$,

$$
\bar{F}(x) \leq M \exp (-t x), \forall x>0 .
$$

Clearly, this links the moment existence directly to the tail behaviour. Hence, one may characterize heavy tailed distributions or processes with impulsive realizations either as 
families which have infinite mean or variance. Or equivalently, via (7), as those distributions which have tails which fail to satisfy this bound for some positive constant $M$.

For instance some of the interference models discussed in Sect. 3.2 (such as the Middleton or the $\alpha$-stable) can be characterized as sub-families of the sub-exponential class on the entire real line. The knowledge of the properties of this class of distributions will allow us to get insight on how one can design receivers that will be robust to a wide range of interference settings.

We denote this sub-exponential class according to the general notation $\mathcal{F}$ (see the original characterization in [84]). This family of heavy tailed distributions has played an important role in many areas of science such as branching phenomena for positive random variables in [85] and insurance contexts in [86]. Here, we introduce it as an overarching model framework for understanding different types of impulsive interference models in wireless communications, where existing models previously discussed can be considered as subfamilies in $\mathcal{F}$.

The original specification of the $\mathcal{F}$ class of interference distributions involved distributions $F$ with support $F: \mathbb{R}^{+} \mapsto[0,1]$ and is given by Definition 1 .

Definition 1 (Sub-exponential Impulsive Noise [87]) The sub-exponential family of distributions defines a class of heavy tailed severity models that satisfy the limits

$$
\lim _{x \rightarrow \infty} \frac{1-F^{n \star}(x)}{1-F(x)}=n
$$

if and only if,

$$
\lim _{x \rightarrow \infty} \frac{1-F^{2 \star}(x)}{1-F(x)}=2
$$

where $F^{n \star}(x)$ is the n-fold convolution of distribution $F$ with itself.

It was shown by [88] that a distribution is a member of this class $(F \in \mathcal{F})$ if and only if it is long-tailed, i.e., it satisfies

$$
\lim _{x \rightarrow \infty} \frac{1-F(x+y)}{1-F(x)}=1, \forall y \in \mathbb{R}^{+}
$$

The extension of the family of sub-exponential distributions to the entire real-line is required for wireless communications applications. This extension is a relatively recent result, see [89, Section 3.2], therefore we believe it will be highly informative for the wireless communications audience to have these results briefly brought to consideration. In particular it can be shown that an interference model is sub-exponential on the entire real line, denoted by $F \in \mathcal{F}_{\mathbb{R}}$, if and only if it satisfies that $F \in \mathcal{F}$ and $F$ is long-tailed on $\mathbb{R}$ and therefore satisfies (10) and finally that given independent $I \sim G_{1}$ and $N \sim G_{2}$ with $G_{i}=O(F(x))$ as $x \rightarrow \infty$ then one has the total interference in the tails given by

$$
\mathbb{P}[I+N>x, I>g(x), N>g(x)]=o(\bar{F}(x)) \text {, as } x \rightarrow \infty
$$


where $g(x)$ is a function that satisfies that $g(x) \rightarrow \infty$ as $x \rightarrow \infty$ and $O($.$) and o($.$) are the$ big-Oh and little-Oh Landau notations.

To understand how such a general characterization of all interference models can be interpreted and becomes practically useful for things like receiver design, consider the following property: consider the noise plus interference distribution in (3) required for receiver design. The impulsive noise term $I$ has a distribution $F_{I}$ in the sub-exponential class. It can be shown that it dominates the Gaussian thermal noise $N$, with distribution $F_{N}$, in the tails. Therefore, one can avoid to calculate any complicated convolutions and instead work with approximation of the heavy tailed interference. Put another way, if one considers the distribution of the maximum, for sub-exponential models one obtains

$$
\mathbb{P}(\max \{I+N\}>x)=\bar{F}_{I}(x) \bar{F}_{N}(x) \sim 2 \bar{F}_{I}(x), \text { as } \mathrm{x} \rightarrow \infty .
$$

where $\sim$ denotes the asymptotic equivalence between the distributions. The equivalent result can be obtained for the minimum also, i.e., the left tail. This property implies that the partial sum is likely to get large when one of the random variables gets large. Importantly, for receiver design such a result tells us that asymptotically the interference distribution dominates so that we need to design receivers flexible enough to adapt to the interference tail behaviour, when receivers based on the Gaussian model are not producing acceptable performance in the presence of impulsive noise because they do not handle the tail behaviour characteristic; this remark is general for all sub-exponential models.

Considering this general overarching characterization of impulsive interference models, we can state that a simple Gaussian approximation for the receiver design will fail to capture the tail behavior and, consequently, will not perform efficiently in practice. We therefore conclude that we must consider a new paradigm for receiver design if we truly wish to have a receiver strategy that is flexible enough to accommodate a variety of different impulsive noise environments and to be adaptive enough to work efficiently in all situations.

\subsection{Receiver design}

When it comes to receiver design, the first observation is the poor behaviour obtained by the linear receiver, which is optimal in a Gaussian noise but highly suboptimal in other interference settings. The second observation is the difficulty in developing an optimal receiver. One reason is the variety of proposed interference models: which model should I design my receiver for and how will it perform if my environment changes? If empirical models, chosen to offer analytical solutions, are attractive, their ability to adapt to different contexts is to be proven. Another reason is that implementing a receiver can be complex for some specific interference distributions, for instance with the infinite series from Middleton's model, stochastic geometry or the absence of closed-form $\alpha$-stable PDF.

\subsubsection{Impact of impulsiveness on the optimal decision}

An efficient way to characterize and understand the influence of impulsive noise is to visualize the impact of the non linearities by representing the decision regions. This was 

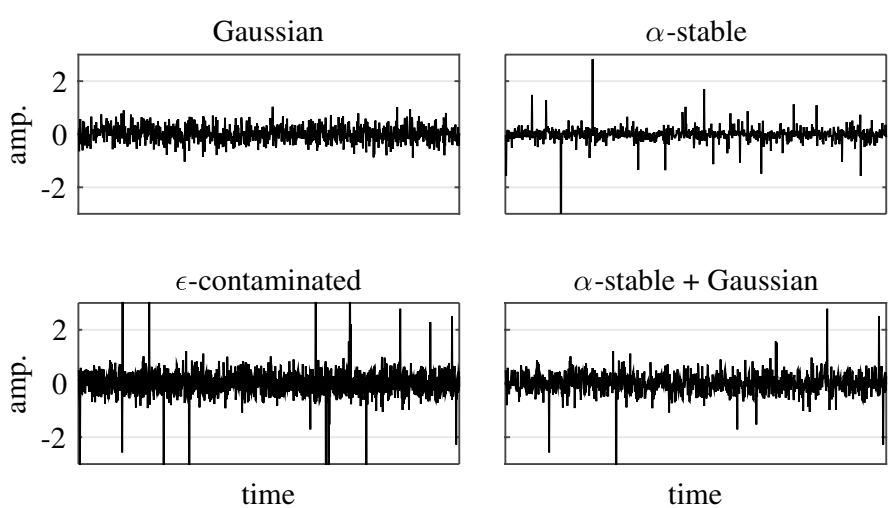

Fig. 1 Realisation examples for different noise processes. The following parameters were used in each case: Gaussian case ( $\mu=0$ and $\left.\sigma^{2}=0.2\right) ; \alpha$-stable $(\alpha=1.5, \gamma=0.1) ; \epsilon$-contaminated case $(\epsilon=0.01, \kappa=100$, $\left.\sigma^{2}=0.2\right)$; sum of Gaussian and $\alpha$-stable in a moderately impulsive case $\left(\alpha=1.5, \gamma=0.1\right.$ and $\sigma^{2}=0.2$ $(N \mid R=0))$

proposed by Saaifan and Henkel [90] for the Middleton class A case and by Shehat et al. [91] and by Saleh et al. [92] for the $\alpha$-stable case.

We represent in Fig. 1 four different examples of noise realizations. Then we show in Fig. 2 the decision regions that the optimal receiver must produce in a binary case under each of the different models, i.e., the regions that maximize the probability of having transmitted $s$ when $\mathbf{Y}=\left(y_{1}, y_{2}\right)$ is received.

The Generalized Gaussian distribution, when the shape parameter is less than 1, is sub-exponential in nature. The Mixture of Gaussians, including the $\epsilon$-contaminated, are not strictly sub-exponential. However, as noted in [93] one can approximate for instance an $\alpha$-stable sub-exponential interference model to an arbitrary accuracy over any tail probabilities, eventually with enough Gaussian mixture components. It is in this context that we consider such models as "impulsive". The $\alpha$-stable distributions belong to the sub-exponential family.

It is well known that the optimal decision regions are linearly separated under interference with exponential tail decay, such as the Gaussian case shown in Fig. 2. However, the optimal decision regions under heavy tailed sub-exponential interference produce nonlinear frontiers and disjoint regions, as seen with the $\alpha$-stable noise. We can identify two operating regions: for small received values $y_{1}, y_{2}$, boundaries are linear. However, when at least one value becomes larger, linear boundaries completely fail to recover the most likely transmitted symbol. The point at which this non-linearities appear is linked to the heaviness of the tail: the heavier it is, the more reduced the linear frontiers are, since the sub-exponential tail asymptotic is dominant sooner.

In the $\epsilon$-contaminated case, we see that for large values, the exponential tail makes the decision boundary become linear again. However, the impulses generated by the rare but large variance Gaussian component in the noise distribution create a non linear area; very similar to the $\alpha$-stable case.

Finally, in the $\alpha$-stable and Gaussian mixture, the heavy-tailed interference noise dominates the light tailed Gaussian thermal noise in extremes and dictates the extent of the 

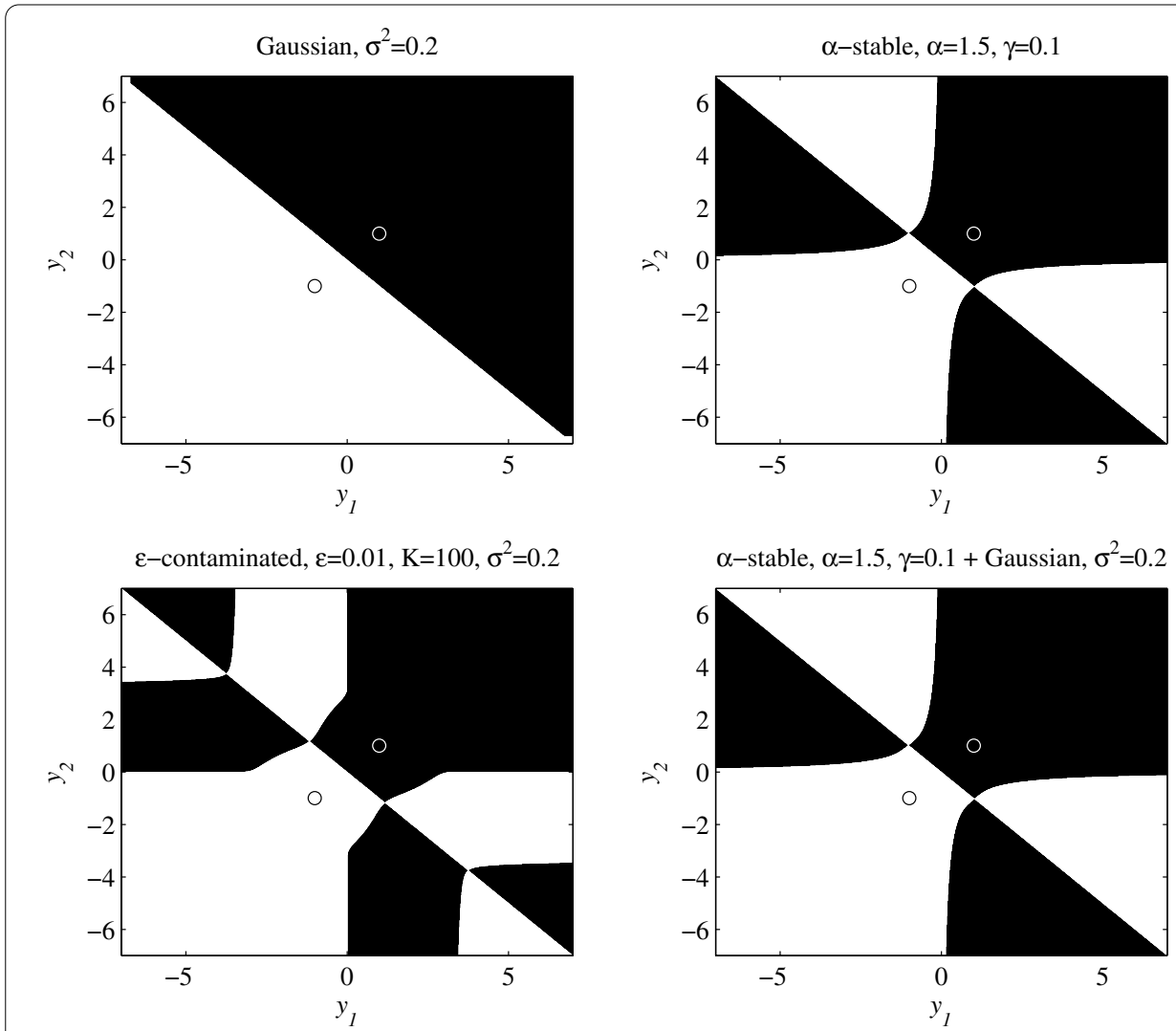

Fig. 2 Optimal decision regions for the different noise processes. We follow the framework proposed in [92] and use the same parameters defined in Fig. 1: the received vector $\mathbf{Y}$ is composed of two received samples (two dimensions, $\mathbf{Y}=\left[\begin{array}{ll}y_{1} & y_{2}\end{array}\right]$ ), the wireless channel is set to $\mathbf{h}=\left[\begin{array}{ll}1 & 1\end{array}\right]$, and we consider two possible transmitted values (ie. $\Omega=\{-1,1\}$ ). The areas in black correspond to a decision $\hat{s}=+1$, the areas in white to $\hat{s}=-1$

non linearity in the decision boundaries, considerably increasing the complexity of the optimal receiver design.

\subsubsection{Overview of receiver strategies}

In the following we do not try to be exhaustive about the existing receiver strategies but we propose to classify the different receiver design approaches into three categories, see Table 2.

\subsubsection{Linear approaches}

Evaluating the LLR with a Gaussian noise assumption results in a linear operation for detection. We primarily consider this choice for its simple implementation structure (and also as a reference), though it is known to perform poorly in impulsive situations. Maximum Ratio Combining maximizes the Signal to Noise Ratio in Gaussian noise. Johnson [94] proposes a general study of linear optimal receivers in non Gaussian noise and takes the specific example of $\alpha$-stable noise. This is further studied for a rake receiver in $[14,95]$ and for diversity combining schemes in a multi-antenna receiver in [96] in presence of symmetric $\alpha$-stable interference. However we found that the improvement 
Table 2 Receiver strategies discussed in this paper

\begin{tabular}{ll}
\hline Receiver strategies & \\
\hline Type of receiver & Examples \\
\hline Linear & Linear combiner [14, 94-96] \\
Noise distribution approximation & Gaussian mixture [97], $\epsilon$-contami- \\
& nated [98], Generalized Gaussian \\
& {$[51]$, mixture of Laplacian and } \\
& Gaussian [50], Cauchy [17], Myriad \\
LLR inspired & {$[20,92,99]$, NIG[23] } \\
& Soft limiter and Hole puncher [66, \\
& $73,92,100,101], p$-norm [105], LLR \\
& approximation $[19]$, approximation \\
& of $f_{l+N}^{\prime}(.) / f_{l+N}().[102-104]$ \\
\hline
\end{tabular}

over the standard linear approach is very limited and we have therefore omitted the corresponding BER curves in Sect. 5.

\subsubsection{Noise distribution approximation}

Another way to solve (4) is to find a distribution that would approximate well the true noise plus interference PDF $f_{I+N}($.) with an analytical expression and parameters that can be simply estimated. If $I$ is sub-exponential and $N$ Gaussian, $I$ will dominate $N$, at least in the tails, so that it is important to increase the heaviness of the tail and several ways have been proposed to do so. Erseghe et al. used a Gaussian mixture for UWB communications [97]. In [98], the $\epsilon$-contaminated is used to study the impact of impulsive noise on Parity Check Codes. The importance to take the real noise model into account during the decoding is underlined. A review in the UWB case can be found in [56]. For instance Fiorina [51] proposed a receiver based on a generalized Gaussian distribution approximation. Beaulieu and Niranjayan [50] considered a mixture of Laplacian and Gaussian noise. The Cauchy model, based on a sub-exponential distribution, is proposed in [17]. Each solution is shown to significantly improve the performance in their specific context. We can wonder how robust they will be in case of a model mismatch.

In this paper, we propose two receivers based on this approach and taking into a account the sub-exponentional nature of the impulsive noise, which means our choices are able to capture the heaviness characteristic of the noise distribution. The Myriad receiver [20,99] is an improved version of the Cauchy receiver [17] for $\alpha \neq 1$ or a mixture of stable and Gaussian interference; as a complement to the work started in [23], we also propose the use of NIG distributions. It is a flexible family of distributions that contains as limiting cases both the Myriad filters and standard linear Gaussian receiver.

Myriad receiver This receiver is based on Cauchy distributions, which are a special case of $\mathrm{S} \alpha \mathrm{S}$ distributions that have an explicit PDF expression with dispersion $\gamma$ and median $\mu$ :

$$
f_{1}(x)=\frac{\gamma}{\pi\left[\gamma^{2}+(x-\mu)^{2}\right]} .
$$




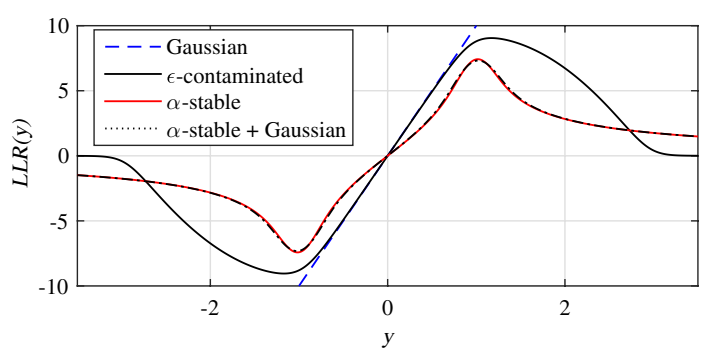

Fig. 3 LLR for the different noise processes. Gaussian case ( $\mu=0$ and $\left.\sigma^{2}=0.2\right) ; \alpha$-stable $(\alpha=1.5, \gamma=0.1)$; $\epsilon$-contaminated case $\left(\epsilon=0.01, \kappa=100, \sigma^{2}=0.2\right)$; sum of Gaussian and $\alpha$-stable in a moderately impulsive case $\left(\alpha=1.5, \gamma=0.1\right.$ and $\left.\sigma^{2}=0.2(N / R=0)\right)$

To improve the adaptability of a receiver based on the Cauchy distribution, the myriad filters have been discussed in $[20,92,99]$. They are based on the Cauchy density but with a modified dispersion parameter $\kappa$ replacing $\gamma$ in (13) so that the decision rule becomes:

$$
\sum_{k=1}^{K} \log \frac{\kappa^{2}+\left(y_{k}+h_{k}\right)^{2}}{\kappa^{2}+\left(y_{k}-h_{k}\right)^{2}} \underset{\widehat{s}=-1}{\stackrel{\widehat{s}=1}{\gtrless} 0 .}
$$

The so called "linearity parameter" $\kappa$ was firstly used to adapt the receiver to interference with an $\alpha$-stable distribution for $\alpha \neq 1$. We discuss the estimation of $\kappa$ in Sect. 4.2.

Normal Inverse Gaussian receiver family We propose the family of receivers specified by the Normal-inverse-Gaussian distributions (details about these distributions are given in "Appendix"). We implement a Symmetric NIG receiver, presented in details in Sect. 4.1. The NIG distribution is denoted $f_{\mathrm{NIG}}(x ; \alpha, \beta, \mu, \delta)$. We note that extension of the receiver can easily be developed for asymmetric cases.

\subsubsection{LLR inspired solutions}

When noise is impulsive with a sub-exponential distribution, the optimal LLR is no longer an monotonic increasing function but tends to reduce the weight of large values in the decision, as shown in [19] in the case of $\alpha$-stable distributions. It means that we should not trust large positive or negative received values, contrary to the decision weight that the linear receiver would attribute. This is illustrated in Fig. 3, which represents the LLR as a function of the received value for the four previously described noise settings.

Except for the Gaussian noise whose LLR is a linear function, the three other cases reaches a maximum and then decrease and tends towards zero. Once again we notice the strong resemblance between the pure $\alpha$-stable and the mixture with the Gaussian noise, curves being nearly identical when superimposed on each other.

This idea leads to a modification of the LLR function and classical examples are the soft limiter and the hole puncher [66, 73, 92, 100, 101]. For small received samples, a linear function is used and for large samples, respectively, a constant value or a zero are used as output of the LLR function. Another approximation is given by: 


$$
\operatorname{LLR}(y)=\operatorname{sign}(y) \min \left(a|y|, \frac{b}{|y|}\right)
$$

where $\operatorname{sign}(x)$ is the sign of $x$. It was proposed in [19] for Low Density Parity Check codes. The model fits the linear part of the LLR for small values of $x$ and the $1 / x$ approximation is inspired from the limit of the likelihood ratio for high values of $x$ in the $\alpha$ -stable case. Parameters $a$ and $b$ are estimated with different methods. Good results are obtained in $\alpha$-stable and Middleton class A interferences.

Other works for weak signal detection approximate the function $f_{I+N}^{\prime}(.) / f_{I+N}($.$) where$ $f^{\prime}($.) is the derivative of $f($.). Zozor et al. [102] for instance used a polynomial approximation of the function. Spaulding and Middleton [103, 104] proposed optimal and suboptimal strategies for coherent and non coherent detection in Middleton Noises. In the coherent case, the optimal detector necessitates to evaluate a ratio of infinite sums, too complex to be implemented. A locally optimum detector is proposed, using a series expansion for small signal. It results in applying a logarithm to the received signal followed by the linear operation.

Another way to analyse detection is to consider that the likelihood measures a distance between the received signal and the possible transmitted signals. Optimal in Gaussian noise, the Euclidean distance is not adapted to the impulsive case. To improve the performance, a solution is then to modify this metric and to use the $p$-norm, which is a distance measurement in $\alpha$-stable situations with $p<\alpha$, see [105],

$$
\|X-Y\|_{\alpha}=\left\{\begin{array}{l}
{\left[\mathbb{E}|X-Y|^{p} / C(\alpha, p)\right]^{1 / p}, 1 \leq \alpha \leq 2} \\
{\left[\mathbb{E}|X-Y|^{p} / C(\alpha, p)\right]^{\alpha / p}, 0<\alpha<1}
\end{array}\right.
$$

where $C(\alpha, p)=\frac{2^{p+1} \Gamma((p+1) / 2) \Gamma(-p / \alpha)}{\alpha \sqrt{\pi} \Gamma(-p / 2)}$, and $\Gamma($.$) is the gamma function. In [106], an inter-$ ference suppression scheme for DS-CDMA systems in the presence of additive S $\alpha \mathrm{S}$ interference is proposed based on the $L_{p}$-norm instead of the standard Least Mean Square based on the $L_{2}$-norm.

This expression is of interest as it does not depend on any estimation of distribution parameters and a rough knowledge of $\alpha$ can be sufficient if the condition $0<p<\alpha$ is fulfilled. We can therefore utilize the $p$-norm metric in our decision statistic as,

$$
\Lambda_{p}(\mathbf{Y})=\sum_{k=1}^{K}\left(\left|y_{k}+h_{k}\right|^{p}-\left|y_{k}-h_{k}\right|^{p}\right) \underset{\widehat{s}=-1}{\stackrel{\widehat{s}=1}{\gtrless} 0 .}
$$

We can notice that this metric would be optimal in a Generalized Gaussian noise.

We present in Fig. 4 the proposed approximations: $p$-norm, soft limiter and hole puncher and from (15).

We see that the $p$-norm and the solution from (15) offer a wider flexibility. The $p$-norm can also mimic the linear case when $p=2$. This aspect is confirmed in the performance evaluation that is why we are not going to represent the performance of the holepuncher nor the soft-limiter. 


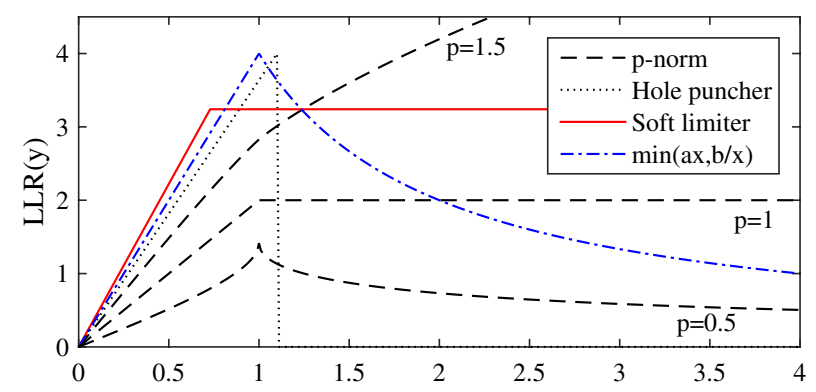

Fig. 4 Examples of approximated LLR
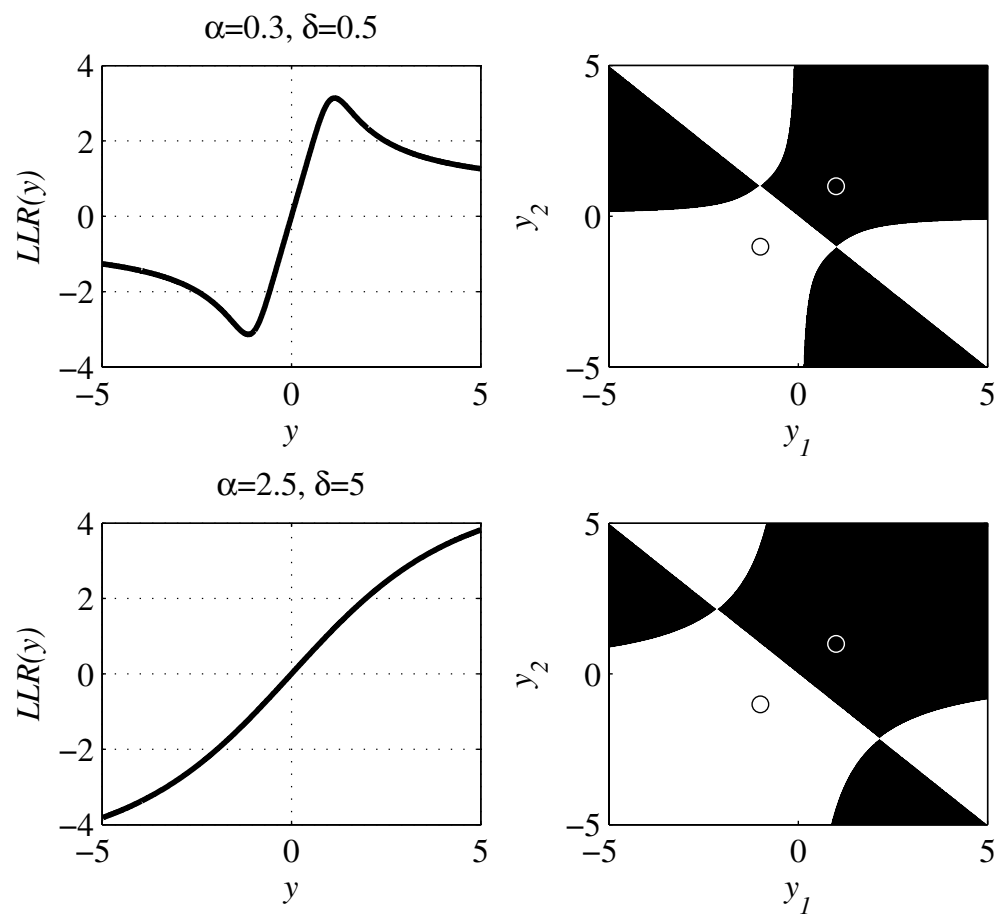

Fig. 5 LLR and decision regions for the NIG receiver when $\alpha=0.3, \delta=0.5$ or $\alpha=2.5, \delta=5(\beta=\mu=0)$

\section{Proposed robust receivers}

In this section we first detail the NIG receiver and the necessary parameters' estimation. We then propose estimation procedures for the parameters $\kappa$ in the Myriad, $p$ in the $p$-norm receivers and $a$ and $b$ for the LLR approximation.

\subsection{Receiver based on the Normal Inverse Gaussian approximation}

In order to develop a flexible receiver that can accommodate high performance in a wide variety of impulsive interference environments, as characterized by the sub-exponential family, we need a receiver which has three practical characteristics: (1) it should have heavy tailed properties commensurate with the sub-exponential characterization in Sect. 3.3; (2) it should be easy to perform online real time estimation the receiver parameters; and (3) wide range of skew-kurtosis property for the density. We will show 
below that the family of distributions known as the NIG model has these three desirable characteristics.

In particular, estimation via Method of Moments is trivially achieved in general for NIG models if one restricts to a subfamily of the NIG distributions, through constraining on the existence of the first four cumulants [107] (see "Appendix", (24)-(27) for the moment expression). The expressions for the parameters of the NIG distribution are then given in (18).

Method of moments closed form parameter estimation for NIG models Given i.i.d. distributed $\operatorname{NIG}(\alpha, \beta, \mu, \delta)$ random variables, the sample mean, variance, skewness and excess kurtosis, denoted by $\hat{\mathcal{M}}, \hat{\mathcal{V}}, \hat{\mathcal{S}}$ and $\hat{\mathcal{K}}$ respectively can be utilized to estimate the model parameters with a constraint imposed. Assume that the following constraint applies to the kurtosis $3 \hat{\mathcal{K}}>5$ and the skewness $\hat{\mathcal{S}}^{2}>0$, then the method of moments estimators for the parameters are given by

$$
\begin{aligned}
& \hat{\alpha}=3 \hat{\rho}^{1 / 2}(\hat{\rho}-1)^{-1} \hat{\mathcal{V}}^{-1 / 2}|\hat{\mathcal{S}}|^{-1}, \\
& \hat{\beta}=3(\hat{\rho}-1)^{-1} \hat{\mathcal{V}}^{-1 / 2} \hat{\mathcal{S}}^{-1}, \\
& \hat{\mu}=\hat{\mathcal{M}}-3 \hat{\rho}^{-1} \hat{\mathcal{V}}^{1 / 2} \hat{\mathcal{S}}^{-1}, \\
& \hat{\delta}=3 \hat{\rho}^{-1}(\hat{\rho}-1)^{1 / 2} \hat{\mathcal{V}}^{1 / 2}|\hat{\mathcal{S}}|^{-1},
\end{aligned}
$$

where $\hat{\rho}=3 \hat{\mathcal{K}} \hat{\mathcal{S}}^{-2}-4>1$.

We can further simplify these expressions for the Symmetric case $(\beta=\mu=0)$. This results in simpler parameter estimators given by $\hat{\alpha}=\sqrt{3} \hat{\mathcal{V}}^{-1 / 2} \hat{\mathcal{K}}^{-1 / 2}$ and $\hat{\delta}=\sqrt{3} \hat{\mathcal{V}}^{1 / 2} \hat{\mathcal{K}}^{-1 / 2}$.

Remark 1 We note that special care has to be taken due to the high order moment calculation, especially in the illustration we take involving stable distribution for the true impulsive interference distribution. To ensure the validity of the obtained parameters, the training data has to respect some restrictions. Another way is to reduce the impact of large samples in the training sequence by a soft thresholding method known widely in statistics as tempering the empirical distribution of the data before calculating the moments. This can also be known as exponential tilting and it ensures the approximate NIG receiver model is always well defined, see discussions in [108].

To illustrate how flexible the proposed NIG receiver behaves in an impulsive noise environment, we plot in Fig. 5 the decision regions as in Sect. 3.2, considering the maximisation problem in (2) with the density $f_{\mathrm{NIG}}(x ; \alpha, 0,0, \delta)$ given in (22).

We notice that the impact of impulsiveness is well taken into account and modifying the parameters will allow to adjust the "linear part" of the receiver. We can expect that this receiver will be able to adjust to different impulsiveness degrees approximating well a wide variety of sub-exponential impulsive noise models, such as those illustrated in Fig. 1.

\subsection{Estimation steps for the Myriad filter}

The myriad filter relies on one parameter $\kappa$. An empirical estimator $\left(\hat{\kappa}_{\alpha}=\sqrt{\frac{\alpha}{2-\alpha}} \gamma^{1 / \alpha}\right)$ was developed in [20] for an $\alpha$-stable noise. It was further modified by Niranjayan and 
Beaulieu [99] in the case of a sum of $\alpha$-stable and Gaussian noises. However, these solutions necessitate to estimate the noise parameters which is not trivial. Consequently we have chosen to directly estimate $\kappa$. This can be done by maximizing the LLR $\lambda_{0}$ given by (we consider the case of a symmetric distribution so that $\mu=0$ ):

$$
\begin{aligned}
\lambda_{0} & =\log f_{I+N}(x)=\sum_{i=1}^{N} \log \left(f_{I+N}\left(x_{i}\right)\right) \\
& =\sum_{i=1}^{N} \log \left(\frac{\kappa}{\pi\left[\kappa^{2}+x^{2}\right]}\right) .
\end{aligned}
$$

where $x=\left(x_{1}, x_{2}, \ldots, x_{N}\right)$ are the i.i.d thermal noise plus interference samples $\left(x_{i}=N_{i}+I_{i}\right)$ which can be obtained when the source is silent. $\kappa$ can be obtained through the derivative by solving:

$$
\frac{\mathrm{d} \lambda_{0}}{\mathrm{~d} \kappa}=0 \Leftrightarrow \sum_{i=1}^{N}\left(\frac{\kappa^{2}}{x_{i}^{2}+\kappa^{2}}\right)-\frac{N}{2}=0,
$$

and $\kappa^{2}$ is simply obtained for instance via a simple univariate root search procedure.

\subsection{Estimation steps for the $p$-norm}

To adapt to different contexts, we propose to estimate $p$ using a similar framework as for estimating the shape parameter of a generalized Gaussian distribution based on the maximum likelihood method [109]. We use a univariate root search procedure method to find the $p$ value that verifies [110]:

$$
1+\frac{\psi(1 / p)}{p}-\frac{\sum_{i=1}^{N}\left|x_{i}\right|^{p} \log \left|x_{i}\right|}{\sum_{i=1}^{N}\left|x_{i}\right|^{p}}+\frac{\log \left(\frac{p}{N} \sum_{i=1}^{N}\left|x_{i}\right|^{p}\right)}{p}=0,
$$

where $\psi($.$) is the digamma function. When the estimated p$ is larger than 2 , we set it to 2 .

\subsection{Estimation for the LLR inspired approach}

We use the approximation given in (15). We will estimate $a$ and $b$ based on a training sequence. We first estimate the probability density function of the noise using a classical kernel based approach. We then estimate the LLR directly by computing (4) using the density estimate. We cannot use the estimated LLR, $L \hat{L} R(x)$, directly to make a decision because of the numerous numerical uncertainties. So we extract from $L \hat{L} R(x)$ the two parameters $a$ and $b$ by considering linear approximations for small values to get $a$ and for the inverse of the large values to get $b$. Defining what are the small and what are the large values remains tricky. We use the empirical rules that small values are $x<h_{i}$ and large values are $x>1.5 h_{i}$ (where $h_{i}$ is the channel attenuation). This leads to good results if we take care of problems due to the division and to the log in (4). When noise is low and for short training sequences, we can still have problems to evaluate one of the two parameters due to missing values. In that case we choose an empirical value that we fixed at two. 
If the proposed approach shows good results, it fails when the noise is too low giving a noise floor when bit error rate reaches approximately $10^{-5}$. This could be improved with more efficient estimation steps.

\section{Simulation results}

An analytical evaluation of our framework is difficult because we want to be flexible on the noise model. Consequently we have preferred to perform extensive simulations to compare the performance of the proposed detection solutions.

The network configuration is set as follows: $K=8$ repetitions are available at the destination. The channels are i.i.d. Rayleigh block fading, with a different coefficient per repetition. We study four representative cases, the same as presented in Fig. 1:

1 A pure Gaussian case; it is important that the proposed receivers behave well when no impulsive noise is present.

2 A pure $\alpha$-stable noise with $\alpha=1.5$; this represents a highly impulsive situation and an interference limited regime where the Gaussian noise is negligible. This can be representative of Network interference as described in [27].

3 A mixture of $\alpha$-stable and Gaussian noises with $\alpha=1.5$ and the noise to interference ratio NIR $=\sigma^{2} /(2 \gamma)=0 \mathrm{~dB}$; this represents a more complex situation with a noise whose density has no explicit form. It is a similar situation as the previous case but the contribution of both impulsive interference and Gaussian thermal noise are significant. The optimal receiver is based on the inverse Fourier transform of the characteristic function.

4. $\epsilon$-contaminated noise with $\epsilon=0.01, \kappa=100$, reflecting rare but strong impulses (highly impulsive noise). This case is not strictly in the sub-Gaussian family and it is interesting to see how receivers will then behave. It is a simplification of the Middleton model thant can be used to model for impulsive noise caused by atmospheric man-made partial discharge, switching effect, electromagnetic interference [35].

We consider four receivers: Gaussian, Myriad, symmetric NIG, $p$-norm and LLRapproximation. They have been selected because they exhibit a good behaviour in the different situations.

In a first step we evaluate the performance of the estimation steps, before comparing the receiving strategies with the BER curves. When the noise involves an $\alpha$-stable impulsive interference, the BER is measured as a function of the inverse dispersion of the $\mathrm{S} \alpha \mathrm{S}$ distributions $(1 / \gamma)$, since the increase of inverse dispersion indicates the decrease of the noise strength, reflecting the conventional signal-to-noise ratio. When the noise is purely Gaussian or $\epsilon$-contaminated, the SNR at the receiver is used for the $x$-axis. The number of training sequence for NIG, Myriad, $p$ and LLR-approximation estimations is set to 200 bits per dimension, so 1600 bits.

\subsection{Estimation}

Estimation performance is good, even if it is difficult sometimes to evaluate because we do not know what the optimal values should be. So we mainly study the variability, given by the standard deviation, and the evolution of the BER as a function of the training 
Table 3 Estimated parameters in the mixture of Gaussian and $a$-stable noise $(a=1.5$, $\left.\gamma=0.3, \sigma^{2}=0.6(N I R=0)\right)$

\begin{tabular}{|c|c|c|c|c|c|}
\hline \multirow[t]{2}{*}{ L } & \multicolumn{2}{|l|}{ Myriad } & \multicolumn{3}{|l|}{ NIG } \\
\hline & $\kappa$ & BER & $a$ & $\gamma$ & BER \\
\hline 40 & $0.412 \pm 5.8 e^{-2}$ & 1.7 & $1.1 \pm 0.52$ & $0.64 \pm 0.29$ & 1.4 \\
\hline 80 & $0.413 \pm 4.2 e^{-2}$ & 1.6 & $0.869 \pm 0.48$ & $0.560 \pm 0.35$ & 1.3 \\
\hline 200 & $0.410 \pm 1.8 e^{-2}$ & 1.5 & $0.418 \pm 0.32$ & $0.328 \pm 0.14$ & 1.4 \\
\hline 1000 & $0.410 \pm 1.3 e^{-2}$ & 1.5 & $0.273 \pm 0.23$ & $0.245 \pm 0.14$ & 2.3 \\
\hline \multirow[t]{2}{*}{ L } & \multicolumn{2}{|l|}{$p$-norm } & \multicolumn{3}{|l|}{ LLR } \\
\hline & $p$ & BER & $a$ & $b$ & BER \\
\hline 40 & $1.42 \pm 0.47$ & 7.9 & $2.68 \pm 1.3$ & $4.1 \pm 0.96$ & 2.3 \\
\hline 80 & $1.280 \pm 0.41$ & 6.4 & $3.27 \pm 1.06$ & $4.93 \pm 0.80$ & 1.6 \\
\hline 200 & $1.05 \pm 0.23$ & 4.0 & $4.13 \pm 0.35$ & $6.27 \pm 1.06$ & 1.2 \\
\hline 1000 & $0.99 \pm 0.17$ & 1.7 & $4.2 \pm 0.24$ & $7.03 \pm 0.96$ & 1.0 \\
\hline
\end{tabular}

The value mean \pm standard deviation is given for the estimated parameters. The BER has to be multiplied by $10^{-3}$

sequence length. We only present results for the Gaussian and Stable mixture in Table 3 and similar tendency are obtained for the other noises.

- $p$-norm receiver the estimator converges but tends to over estimate $p$ when the training sequence is short and the estimation exhibits an important variability, which degrades the BER. A long training sequence is necessary to reach the optimal performance

- Myriad receiver the estimator of $\kappa$ is certainly the most robust one. The standard deviation of the estimated value is very low, even for short training sequences and the mimimum BER is rapidly reached.

- NIG receiver values of the parameters, as well as the standard deviation, decrease when the training length increases. However the lower BER is reached for rather short training sequences ( $\sim 100$ bits) but increases with longer training sequences. This is due to the difficult estimation of the higher order moments in sub-exponential noises. Longer sequences increase the risk of restriction violation (see Remark 1) in the estimation process, inducing the error degradation.

- LLR-approximation: It exhibits a large variability in the estimated parameters as the training sequence length increases. Long sequences are necessary to reach low BER. However, it is the one reaching the lowest BER. The estimation procedure in that case has to be optimized.

In the following, we take a long training sequence (1600 bits), ensuring the convergence of the algorithms. Shorter sequences may modify the optimal receiver choice. The noise approximations work better when the training sequence is short and rapidly exhibit good performance. The LLR approximations converge much slower but reaches better performance for long training sequence and, as seen in Fig. 8 attains the optimal performance. 


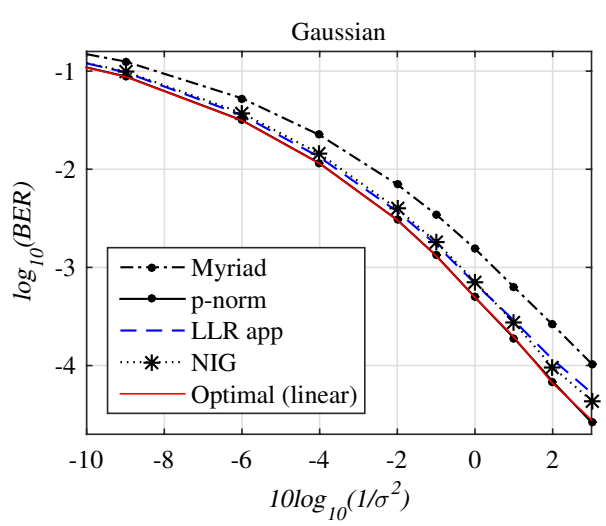

Fig. 6 BER comparison of different receivers, Gaussian noise

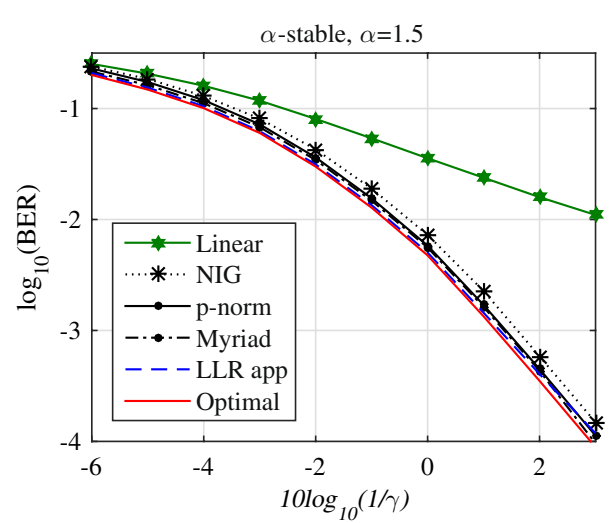

Fig. 7 BER comparison of different receivers, impulsive environment

\subsection{Performance in Gaussian noise}

In Fig. 6, the linear combiner (maximum ratio combining) is the optimal receiver. The $p$-norm receiver reaches the same performance as the optimal one, meaning that in that case the estimation of $p$ works well, always giving values close to 2 , which corresponds to the optimal receiver. The values slightly less than two do not introduce errors and we have set to 2 estimated values larger than 2 .

The NIG and LLR approximation perform very close to the optimal. Only the Myriad receiver exhibits a slight degradation of the performance. This is naturally explained because it is based on a modified version of the Cauchy distribution [see (13) and (14)] and the receiver has difficulties to well behave in noises with exponential tails.

\subsection{Performance in $\alpha$-stable noise}

In Fig. 7, $\alpha$ is set to 1.5 , corresponding to a rather impulsive noise. All receivers perform well but the linear receiver, which exhibits a very significant performance degradation. This last solution is indeed badly affected by large, but rare, values. The four other proposals ( $p$-norm, Myriad, NIG, LLR approximation) behave well and very close to the optimal solution. 


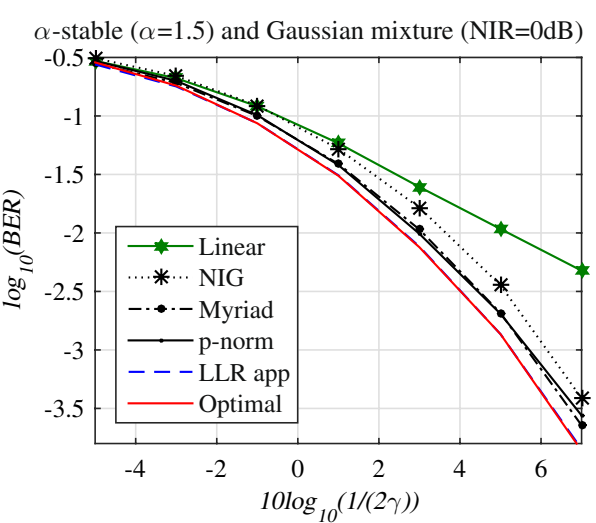

Fig. 8 BER comparison of different receivers, mixed stable and Gaussian environment

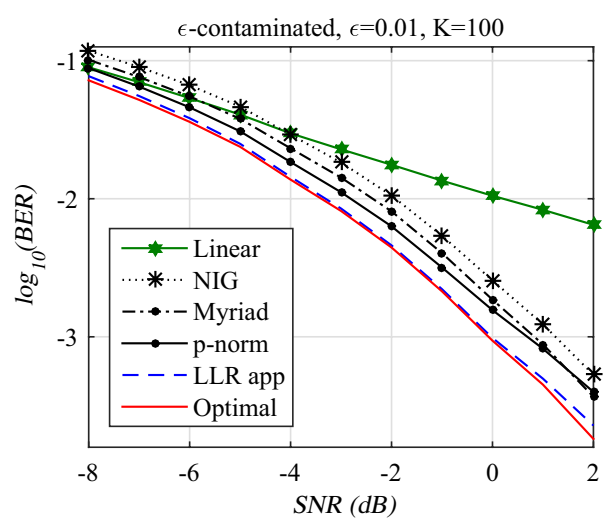

Fig. 9 BER comparison of different receivers, $\epsilon$-contaminated noise

\subsection{Performance in a mixture of $\alpha$-stable and Gaussian noise}

In Fig. $8, \alpha$ is set to 1.5 for network interference and NIR is $0 \mathrm{~dB}$. This case includes both a sub-exponential type component and a more traditional thermal noise. Once again the linear receiver can not handle large received noise samples and its performance are significantly degraded.

The four other solutions behave well, close again from the optimal. The LLR approximation is in this case very close to the optimal. In the LLR, the linear part corresponds to the Gaussian noise for small values and the $1 / x$ part to the tails of the distribution which is dominated by the sub-exponential contribution.

We notice in the example that, as suggested by the discussion about (12), the subexponential component dictates the tail behaviour and it is important that the receiver takes this into account.

\subsection{Performance in $\epsilon$-contaminated noise}

Finally we consider in Fig. 9 the $\epsilon$-contaminated environment with epsilon $=0.01$ and $\kappa=100$. This situation represents a highly impulsive noise but without a heavy tail representation. Again, the linear receiver sees its performance far below the optimality 
when the others tend to get very close. The LLR approximation is once again the most efficient approach when the NIG exhibits a slight loss.

\subsection{Summary}

From the preceding figures, and also considering other approaches we did not include here, we make the following comments:

1 Linear receivers only behave well in Gaussian noise. When an impulsive component is present, the degradation in comparison to receivers that take this component into account is significantly poorer as seen in Fig. 8. This is especially clear when the noise is purely sub-exponential (Fig. 7) but it is also true with models having exponential tails, like the $\epsilon$-contaminated in Fig. 9 when rare but large samples are present. This conclusion would be similar, even if optimal linear receivers [94] were considered.

2 Approaches trying to approximate the noise distribution give good performance. We evaluated two flexible families of distributions with parameters that can be easily and efficiently estimated: (a) the Myriad with a single parameter to be estimated with a quick and efficient root search; $(b)$ the NIG distribution family presents both the required flexibility and an easy parameter estimation procedure based on moment estimation. Both approaches are robust and adaptive but the NIG outperforms the myriad in Gaussian noise. For short training sequences they are more efficient than LLR based approaches, due to efficient estimation algorithms. It would be needed to further study the complexity issue, especially for the NIG receiver that relies on more complex function, including a Bessel function.

3 LLR approximation based approaches have a good potential. They have been less studied in the literature and more work has to be done in that direction. The intuitive approaches are the soft limiter and the hole puncher that limits the impact of the large values. If they improve the performance in comparison to the linear approach when impulsive interference is present and have a limited impact when impulsiveness decreases, their performance remains far from the optimal [101] and we did not include them in our result section. We studied two other alternatives. If they increase the complexity, they significantly outperform the soft limiter. The $p$-norm allows either a "close to linear" or linear behaviour when the Gaussian noise is the main contribution to the noise and also approaches the sharp shape of the LLR when impulsiveness increases. A single parameter has to be estimated with a root search numerical procedure. The LLR-approximation is also very efficient. It is the receiver that adapts the best to configuration mixing different distributions. Especially the linear part adapt to low noise value corresponding to the Gaussian part and the $1 / x$ part correspond to the tail of the interference, dominated by the sub-exponential component of the total noise. It is however more complex to estimated but recent proposals allow to address this task $[25,111]$.

It is important to consider the non Gaussian nature of the noise in the receiver design. Having in mind the sub-exponential class of distribution can give hints for the design of a receiver, either by approaching the noise distribution or approximating the LLR. If both approaches tend to give close results, some research questions are still opened. 
Complexity is an issue but implementation of each solution has to be optimized. We have shown that the receiver can be defined by a limited set of parameters. This is especially true for the $p$-norm and the myriad filter which only require a single parameter. Besides, making assumptions on the noise distribution allows one to rely on well-studied algorithms for the parameter estimation, ensuring an accurate result with a shorter training sequence.

Adaptability and robustness to different situations are also important. For instance, if the Myriad filter seems to be very efficient, performance is not so good when the noise is just Gaussian. And this could happen when the activity in the network is varying with time. LLR approximation and $p$-norm seem very flexible and attractive but require a longer training sequence. However, the estimation has not been optimized and further research is needed, as proposed in [111] for instance.

\section{Conclusion}

Interference is a significant limiting factor in many communication situations. Besides it is not Gaussian distributed in many cases. A large amount of papers have dealt with such contexts for many different physical layers and many different applications and the topic is still very active.

We proposed in this paper a study of impulsive noise models. We defined impulsive interference thanks to a broad class of models, the sub-exponential family, and showed the impacts interference has on the optimal signal detection regions, resulting in non linear decision boundaries, not even contiguously joined. To design receivers, we proposed to classify the different approaches (linear, flexible density functions, LLR-inspired). Based on it, we evaluated the $p$-norm, the NIG, the Myriad and a LLR-approximation receivers. We included the estimation steps of the receivers and tested them in different contexts, modifying the impact of impulsiveness and the models for interference. The four solutions under test outperform the traditional linear approach. They offer simple parameter estimation and robustness in environments that are impulsive or not.

The wireless communication environment has significantly changed in the last decades. However engineers are generally still using the Gaussian assumption for receiver designs. This is significantly sub-optimal in many cases. The difficult point is that the receiver has to adapt to many different scenarios depending on space and time and the transmission conditions are generally unpredictable. We showed with only one or two parameters, a robust and adaptive receiver can be designed but it is still to be implemented in real systems and the impact of the hardware part is also to be studied and probably adapted. Another important issue is to model the space, time and frequency dependence [27] of the interference and to link it with the resource allocation strategy [112] to improve the scalability and coexistence of networks.

\section{Abbreviations}

BER: Bit error rate; CDMA: Code division multiple access; CF: Characteristic function; DS-CDMA: Direct sequence code division multiple access; LLR: Log likelihood ratio; ML: Maximum likelihood; MGF: Moment generating function; NIG: Normal Inverse Gaussian; OFDM: Orthogonal frequency division multiplexing; PDF: Probability density function; RV: Random variable; TH-UWB: Time hopping ultra wide band; UWB: Ultra wide band. 


\begin{abstract}
Authors' contributions
All the authors fully contributed to this papers through many discussions and collaborative works. They all participated to the writing. We can detail slightly the contribution of each author although all parts were really joint works. LC especially developed the receiver design and state of the art and performed the simulations. GWP proposed the sub-exponential and NIG approaches. FS proposed the parameter estimation approaches. IN contributed to the wireless communication problem formalization and the Maximum Likelihood development. All authors read and approved the final manuscript.

Funding

The work is supported by the french ANR project ARBURST.

Availability of data and materials

Data sharing not applicable to this article as no datasets were generated or analysed during the current study. All results are from simulations that can be easily reproduced.

Competing interests

The authors declare that they have no competing interests.

Author details

'IMT Lille Douai, Univ. Lille, CNRS, UMR 8520 - IEMN, 59000 Lille, France. ${ }^{2}$ Department of Actuarial Mathematics and Statistics, Heriot-Watt University, Edinburgh, UK. ${ }^{3}$ University Bretagne Sud / Lab-STICC UMR CNRS 6285, Vannes, France.

${ }^{4}$ TUM CREATE, 1 Create Way, Create Tower, Singapore, Singapore.
\end{abstract}

\title{
Appendix: NIG distribution
}

The NIG distributional family is characterized by four parameters $\alpha, \beta, \mu$ and $\delta$ (the same letters as the stable family which are used in a similar manner): $\alpha$ is inversely related to the heaviness of the tails, where a small $\alpha$ corresponds to heavy tails that can accommodate outlying observations; skewness is directly controlled by the parameter $\beta$, where negative (positive) values of $\beta$ result in a left (right) skew, and $\beta=0$ is the symmetric model; location (or translation) of the distribution is given by the parameter $\mu$; scale of the distribution is given by the parameter $\delta$. Notably, when $\beta=0$ and $\mu$ is arbitrary, the NIG model asymptotically approaches the Gaussian model $X \sim \mathcal{N}\left(\mu, \frac{\delta}{\alpha}\right)$ as $\alpha \rightarrow \infty$. Hence one could approximate the optimal linear receiver when only Gaussian thermal noise is incident on the received signal. In addition, when $\alpha=\beta=0$ with $\mu$ and $\delta$ arbitrary, the NIG model approaches the Cauchy distribution. It can also approximate the skewness and kurtosis of the log-normal, Student's $t$, and gamma distributions, among others [113].

The NIG model takes its name from the fact that it represents a normal variance-mean mixture that occurs as the marginal distribution for a random variable $X$ when considering a pair of random variables $(X, Z)$ where $Z$ is distributed as an inverse Gaussian $Z \sim I G\left(\delta, \sqrt{\alpha^{2}-\beta^{2}}\right)$, and $X$ conditional on $Z$ is $(X \mid Z=z) \sim N(\mu+\beta z, z)$, see [114]. The resulting density function is given in the following definition:

Definition 2 (Normal Inverse Gaussian Density) A random variable $X \sim \operatorname{NIG}(\alpha, \beta, \mu, \delta)$ is characterized by the density function

$$
f_{\mathrm{NIG}}(x ; \alpha, \beta, \mu, \delta)=\frac{\alpha \delta}{\pi} \frac{\exp (g(x))}{h(x)} K_{1}(\alpha h(x)),
$$

where the functions $g(\cdot)$ and $h(\cdot)$ are defined as $g(x)=\delta \sqrt{\alpha^{2}-\beta^{2}}+\beta(x-\mu)$, and $h(x)=\left[(x-\mu)^{2}+\delta^{2}\right]^{1 / 2}$ and $K_{1}[\cdot]$ is a modified Bessel function of the second (or third) kind with index 1 . The parameters have the constraints $\mu \in \mathcal{R}, \delta>0,0 \leq|\beta| \leq \alpha$.

We can study the distribution function tail behaviour of the NIG receiver model, showing that indeed it satisfies the properties required for sub-exponential models. 
Property 1 Consider a NIG random variable. The tail behaviour for the density and distribution functions can be characterized asymptotically as $x \rightarrow \infty$ as follows:

$$
\begin{aligned}
& f_{N I G}(x ; \alpha, \beta, \mu, \delta) \sim|x-\mu|^{-3 / 2} \exp (-\alpha|x-\mu|+\beta(x-\mu)) \\
& \mathbb{P}(X-\mu>x) \sim x^{-3 / 2} \exp (-(\alpha-\beta) x) \\
& \mathbb{P}(X-\mu<-x) \sim x^{-3 / 2} \exp (-(\alpha+\beta) x)
\end{aligned}
$$

These asymptotics then show that indeed the NIG receiver model can be shown to be long-tailed and therefore an admissible sub-family of the subexponential impulsive interference models. To see this we consider the definition given in (10). We apply it to the NIG case and see that the asymptotic of the numerator for any $y \in \mathbb{R}^{+}$is given by considering for instance the right tail asymptotic ratio

$$
\begin{aligned}
& \lim _{x \rightarrow \infty} \frac{1-F(x+y)}{1-F(x)} \\
& \quad=\lim _{x \rightarrow \infty} \frac{(x+y)^{-3 / 2} \exp (-(\alpha-\beta)(x+y))}{x^{-3 / 2} \exp (-(\alpha-\beta) x)} \\
& \quad=\exp ((\beta-\alpha) y) .
\end{aligned}
$$

Hence, if one sets $\alpha=\beta$ then the resulting distribution has limit of 1 and the receiver model we propose clearly captures the class of sub-exponential models. When $\alpha \neq \beta$, one gets other more general approximations which can also be achieved in addition to heavy tailed sub-exponential interference models, clearly demonstrating the flexibility of the NIG receiver model we propose.

The final characteristic is that it is easily calibrated and can adaptively alter its characteristics to time changing or varying ranges of impulsive interference environment. This means that, in practice, the resulting receiver model is easily estimated online. The ease of estimation arises from the fact that the NIG distributional family has sufficient statistics given by the first four moments (mean, variance, skewness and kurtosis) and the ability to explicitly solve for the parameters in terms of the cumulants of the distribution using Method of Moments:

$$
\begin{aligned}
& \mathbb{E}(X)=\mu+\frac{\delta\left(\frac{\beta}{\alpha}\right)}{\left(1-\left(\frac{\beta}{\alpha}\right)^{2}\right)^{1 / 2}}, \\
& \operatorname{Var}(\mathrm{X})=\frac{\delta}{\alpha\left(1-\left(\frac{\beta}{\alpha}\right)^{2}\right)^{3 / 2}}, \\
& \operatorname{Skew}(\mathrm{X})=\frac{3\left(\frac{\beta}{\alpha}\right)}{(\delta \alpha)^{1 / 2}\left(1-\left(\frac{\beta}{\alpha}\right)^{2}\right)^{1 / 4}},
\end{aligned}
$$




$$
\mathbb{K u r t}(\mathrm{X})=3 \frac{4\left(\frac{\beta}{\alpha}\right)^{2}+1}{\delta \alpha\left(1-\left(\frac{\beta}{\alpha}\right)^{2}\right)^{1 / 2}}
$$

Received: 16 August 2018 Accepted: 26 November 2020

Published online: 22 January 2021

\section{References}

1. Z. Xu, C. Yang, Z. Tan, Z. Sheng, Raptor code-enabled reliable data transmission for in-vehicle power line communication systems with impulsive noise. IEEE Commun. Lett. 21(10), 2154-2157 (2017)

2. G.A. Al-Rubaye, C.C. Tsimenidis, M. Johnston, Improved performance of TC-OFDM-PLNC for PLCs using exact derived impulsive noise pdfs, in IEEE International Conference on Communications Workshops (ICC Workshops), Paris, pp. 1271-1276 (2017)

3. T. Bai, H. Zhang, R. Zhang, L.L. Yang, A.F.A. Rawi, J. Zhang, L. Hanzo, Discrete multi-tone digital subscriber loop performance in the face of impulsive noise. IEEE Access 5, 10478-10495 (2017)

4. U. Epple, M. Schnell, Advanced blanking nonlinearity for mitigating impulsive interference in OFDM systems. IEEE Trans. Veh. Technol. 66(1), 146-158 (2017)

5. M. Mostafa, Stability proof of iterative interference cancellation for ofdm signals with blanking nonlinearity in impulsive noise channels. IEEE Signal Process. Lett. 24(2), 201-205 (2017)

6. N.B. Sarr, A.K. Yazbek, H. Boeglen, J.P. Cances, R. Vauzelle, F. Gagnon, An impulsive noise resistant physical layer for smart grid communications, in IEEE International Conference on Communications (ICC), Paris, France, pp. 1-7 (2017)

7. I. Landa, A. Blazquez, M. Velez, A. Arrinda: Indoor measurements of loT wireless systems interfered by impulsive noise from fluorescent lamps, in 11th European Conference on Antennas and Propagation (EUCAP), Paris, pp. 2080-2083 (2017)

8. Y. Ai, M.A. ChitreCheffena, On multi-hop decode-and-forward cooperative relaying for industrial wireless sensor networks. Sensors 17(4), 695 (2017)

9. P. Chen, Y. Rong, S. Nordholm, Z. He, A.J. Duncan, Joint channel estimation and impulsive noise mitigation in underwater acoustic OFDM communication systems. IEEE Trans. Wireless Commun. 16(9), 6165-6178 (2017). https ://doi.org/10.1109/TWC.2017.2720580

10. A. Mahmood, M. Chitre, Ambient noise in warm shallow waters: a communications perspective. IEEE Commun. Mag. 55(6), 198-204 (2017)

11. S. Liu, F. Yang, W. Ding, J. Song, Double kill: compressive-sensing-based narrow-band interference and impulsive noise mitigation for vehicular communications. IEEE Trans. Veh. Technol. 65(7), 5099-5109 (2016)

12. A.J. Ganesh, G.L. Torrisi, Large deviations of the interference in a wireless communication model. IEEE Trans. Inf. Theory 54(8), 3505-3517 (2008). https://doi.org/10.1109/TIT.2008.926304

13. M. Egan, L. Clavier, M. de Freitas, L. Dorville, J.M. Gorce, A. Savard, Wireless communication in dynamic interference, in IEEE GLOBECOM, Singapore (2017)

14. S. Niranjayan, N.C. Beaulieu, The BER optimal linear rake receiver for signal detection in symmetric $a$-stable noise IEEE Trans. Commun. 57(12), 3585-3588 (2009)

15. M.C. Filippou, D. Gesbert, G.A. Ropokis, Optimal combining of instantaneous and statistical CSI in the SIMO interference channel, in IEEE 77th Vehicular Technology Conference (VTC Spring), pp. 1-5 (2013)

16. J. Chen, L. Clavier, Y. Xi, A. Burr, N. Rolland, P.A. Rolland, Alpha-stable interference modelling and relay selection for regenerative cooperative IR-UWB systems, in European Wireless Technology Conference (EuWiT) (2010)

17. H.E. Ghannudi, L. Clavier, N. Azzaoui, F. Septier, P.-A. Rolland, $a$-stable interference modeling and cauchy receiver for an IR-UWB ad hoc network. IEEE Trans. Commun. 58, 1748-1757 (2010)

18. W. Gu, L. Clavier, N. Rolland, P.A. Rolland, Turbo code decoding in MAl environment, in 6th International Symposium on Turbo Codes \& Iterative Information Processing, Brest, France (2010)

19. V. Dimanche, A. Goupil, L. Clavier, G. Gellé, On detection method for soft iterative decoding in the presence of impulsive interference. IEEE Commun. Lett. 18(6), 945-948 (2014)

20. J.G. Gonzalez, G.R. Arce, Optimality of the myriad filter in practical impulsive-noise environments. IEEE Trans. Signal Process. 49(2), 438-441 (2001). https://doi.org/10.1109/78.902126

21. P. Yang, Y.L. Guan, X.B. Liu, Z. Liu, An improved hybrid turbo equalizer for single carrier transmission with impulsive noise and isi. IEEE Trans. Veh. Technol. 66(11), 9852-9861 (2017)

22. H. Shao, N.C. Beaulieu, Analysis of a novel p-order metric UWB receiver structure with improved performance in multiple access interference, in Proceeding of IEEE Globecom, Washington D.C., pp. 4112-4117 (2007)

23. W. Gu, G. Peters, L. Clavier, F. Septier, I. Nevat: Receiver study for cooperative communications in convolved additive alpha-stable interference plus Gaussian thermal noise, in International Symposium on Wireless Communication Systems (ISWCS), pp. 451-455 (2012). https://doi.org/10.1109/ISWCS.2012.6328408

24. V. Dimanche, A. Goupil, L. Clavier, G. Gellé, Estimation of an approximated likelihood ratio for iterative decoding in impulsive environment, in Fifth Annual Symposium on Operating Systems Design and Implementation, Doha, Qatar, pp. 1-6 (2016) 
25. Y. Mestrah, A. Savard, A. Goupil, L. Clavier, G. Gellé, Blind estimation of an approximated likelihood ratio in impulsive environment, in IEEE 29th Annual International Symposium on Personal, Indoor and Mobile Radio Communications (PIMRC), Bologna, Italy, pp. 1-5 (2018)

26. S. Kharbech, I. Dayoub, M. Zwingelstein-Colin, E.P. Simon, Blind digital modulation identification for mimo systems in railway environments with high-speed channels and impulsive noise. IEEE Trans. Veh. Technol. 67(8), 7370-7379 (2018)

27. M. Egan, L. Clavier, C. Zheng, M. de Freitas, J.M. Gorce, Dynamic interference for uplink scma in large-scale wireless networks without coordination. EURASIP J. Wirel. Commun. Netw. 2018(1), 213 (2018)

28. C. Zheng, M. Egan, L. Clavier, G.W. Peters, J. Gorce, Copula-based interference models for iot wireless networks, in ICC 2019-2019 IEEE International Conference on Communications (ICC), pp. 1-6 (2019)

29. A. Mahmood, M. Chitre, M.A. Armand, PSK communication with passband additive symmetric $a$-stable noise. IEEE Trans. Commun. 60(10), 2990-3000 (2012)

30. K. Furutsu, T. Ishida, On the theory of amlitude distribution of impulsive random noise and its application to the atmospheric noise. J. Radio Res. Lab. 7(32), 279-307 (1960)

31. A.A. Giordano, F. Haber, Modeling of atmospheric noise. Radio Sci. 7, 1011-1023 (1972)

32. D. Middleton, Statistical-physical models of electromagnetic interference. IEEE Trans. Electromagn. Compat. 19(3), 106-127 (1977)

33. D. Middleton, Non-Gaussian noise models in signal processing for telecommunications: new methods and results for class a and class b noise models. IEEE Trans. Inf. Theory 45(4), 1129-1149 (1999)

34. H. Oh, H. Nam, Design and performance analysis of nonlinearity preprocessors in an impulsive noise environment. IEEE Trans. Veh. Technol. 66(1), 364-376 (2017)

35. O. Alhussein, I. Ahmed, J. Liang, S. Muhaidat, Unified analysis of diversity reception in the presence of impulsive noise. IEEE Trans. Veh. Technol. 66(2), 1408-1417 (2017)

36. K.S. Vastola, Threshold detection in narrow-band non-Gaussian noise. IEEE Trans. Commun. 32(2), 134-139 (1984)

37. N. Guney, H. Deliç, M. Koca, Robust detection of ultra-wideband signals in non-Gaussian noise. IEEE Trans. Microw. Theory Tech. 54(4), 1724-1730 (2006)

38. P. Salvo Rossi, D. Ciuonzo, K. Kansanen, T. Ekman, Performance analysis of energy detection for MIMO decision fusion in wireless sensor networks over arbitrary fading channels. IEEE Trans. Wireless Commun. 15(11), 7794-7806 (2016). https://doi.org/10.1109/TWC.2016.2607703

39. S.P. Herath, N.H. Tran, T. Le-Ngoc, On optimal input distribution and capacity limit of Bernoulli-Gaussian impulsive noise channels, in IEEE International Conference on Communications, pp. 3429-3433 (2012)

40. H.V.Vu, N.H. Tran, T.V. Nguyen, S.I. Hariharan, Estimating Shannon and constrained capacities of Bernoulli-Gaussian impulsive noise channels in Rayleigh fading. IEEE Trans. Commun. 62(6), 1845-1856 (2014)

41. D. Fertonani, G. Colavolpe, A robust metric for soft-output detection in the presence of class-a noise. IEEE Trans. Commun. 57(1), 36-40 (2009)

42. B. Nikfar, T. Akbudak, A.J.H. Vinck, MIMO capacity of class A impulsive noise channel for different levels of information availability at transmitter, in 18th IEEE International Symposium on Power Line Communications and Its Applications (ISPLC), pp. 266-271 (2014)

43. E. Axell, P. Eliardsson, S. Tengstrand, K. Wiklundh, Power control in interference channels with class a impulse noise. IEEE Wirel. Commun. Lett. 6(1), 102-105 (2017)

44. D. Fertonani, G. Colavolpe, On reliable communications over channels impaired by bursty impulse noise. IEEE Trans. Commun. 57(7), 2024-2030 (2009)

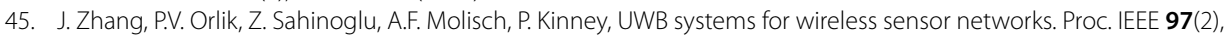
313-331 (2009)

46. G. Durisi, G. Romano, On the validity of Gaussian approximation to characterize the multiuser capacity of UWB TH-PPM, in IEEE Conference on Ultra Wideband Systems and Technologies, pp. 20-23 (2002)

47. A.R. Forouzan, M. Nasiri-Kenari, J.A. Salehi, Performance analysis of time hopping spread-spectrum multiple access system: uncoded and coded schemes. IEEE Trans. Wireless Commun. 1(4), 671-681 (2002)

48. M. Sabattini, E. Masry, L.B. Milstein, A non-Gaussian approach to the performance analysis of UWB TH-BPPM systems, in IEEE Conference on Ultra Wideband Systems and Technologies, pp. 52-55 (2003)

49. G. Durisi, S. Benedetto, Performance evaluation of TH-PPM UWB systems in the presence of multiuser interference. IEEE Commun. Lett. 7(5), 224-226 (2003)

50. N.C. Beaulieu, S. Niranjayan, UWB receiver designs based on a Gaussian-Laplacian noise-plus-MAI model. IEEE Trans. Wireless Commun. 58(3), 997-1006 (2010)

51. J. Fiorina, A simple IR-UWB receiver adapted to multi-user interferences, in IEEE global telecommunications conference, GLOBECOM, pp. 1-4 (2006)

52. N.C. Beaulieu, H. Shao, J. Fiorina, P-order metric UWB receiver structures with superior performance. IEEE Trans. Commun. 56, 1666-1676 (2008)

53. F. Kharrat-Kammoun, C.J. Le Martret, P. Ciblat, Performance analysis of IR-UWB in a multi-user environment. IEEE Trans. Wireless Commun. 8(11), 5552-5563 (2009)

54. B. Hu, N.C. Beaulieu, On characterizing multiple access interference in TH-UWB systems with impulsive noise models, in Proceedings of IEEE Radio Wireless Symposium, Orlando, FL, pp. 879-882 (2008)

55. Z. Mei, M. Johnston, S.L. Goff, L. Chen, Performance analysis of LDPC-coded diversity combining on rayleigh fading channels with impulsive noise. IEEE Trans. Commun. 65(6), 2345-2356 (2017)

56. N.C. Beaulieu, D.J. Young, Designing time-hopping ultrawide bandwidth receivers for multiuser interference environments. Proc. IEEE 97, 255-284 (2009)

57. E.M. Shaheen, Non-Gaussian MAI modeling to the performance of TH-BPSK/PPM UWB communication systems, in 8th International Wireless Communications and Mobile Computing Conference (IWCMC), pp. 916-920 (2012)

58. P.C. Pinto, C.-C. Chong, A. Giorgetti, M. Chiani, M.Z. Win, Narrowband communication in a poisson field of ultrawideband interferers, in The IEEE 2006 International Conference on Ultra-Wideband (ICUWB), pp. 387-392 (2006) 
59. M.Z. Win, P.C. Pinto, A. Giorgetti, M. Chiani, L.A. Shepp, Error performance of ultrawideband systems in a Poisson field of narrowband interferers, in 2006 IEEE Ninth International Symposium on Spread Spectrum Techniques and Applications, pp. 410-416 (2006). https://doi.org/10.1109/ISSSTA.2006.311805

60. A. Rabbachin, T.Q.S. Quek, P.C. Pinto, I. Oppermann, M.Z. Win, UWB energy detection in the presence of multiple narrowband interferers, in IEEE International Conference on Ultra-Wideband, 2007. ICUWB 2007, pp. 857-862 (2007). https://doi.org/10.1109/ICUWB.2007.4381064

61. S. Niranjayan, N.C. Beaulieu, The optimal BER linear rake receiver for alpha-stable noise, in IEEE International Conference on Communications, pp. 5013-5017 (2008)

62. F. Baccelli, B. Blaszczyszyn, Stochastic geometry and wireless networks: volume i theory. Found. Trends Netw. 3(3-4), 249-449 (2010). https://doi.org/10.1561/1300000006

63. F. Baccelli, B. Blaszczyszyn, Stochastic geometry and wireless networks: volume ii applications. Found. Trends Netw. 4(1-2), 1-312 (2010). https://doi.org/10.1561/1300000026

64. S. Weber, J.G. Andrews, Transmission capacity of wireless networks. Found. Trends Netw. 5(2-3), 109-281 (2012). https://doi.org/10.1561/1300000032

65. E.S. Sousa, Performance of a spread spectrum packet radio network link in a Poisson field of interferers. IEEE Trans. Inform. Theory 38(6), 1743-1754 (1992)

66. G.A. Tsihrintzis, C.L. Nikias, M. Shao, Performance of optimum and suboptimum receivers in the presence of impulsive noise modeled as an alpha-stable process. IEEE Trans. Commun. 43(2), 904-914 (1995)

67. J. Ilow, D. Hatzinakos, Analytic alpha-stable noise modeling in a poisson field of interferers or scatterers. IEEE Trans. Signal Process. 46(6), 1601-1611 (1998)

68. H. Inaltekin, S.B. Wicker, M. Chiang, H.V. Poor, On unbounded path-loss models: effects of singularity on wireless network performance. IEEE J. Sel. Areas Commun. 27(7), 1078-1092 (2009)

69. P. Cardieri, Modeling interference in wireless ad hoc networks. IEEE Commun. Surv. Tutor. 12(4), 551-572 (2010). https://doi.org/10.1109/SURV.2010.032710.00096

70. M. Di Renzo, P. Guan, A mathematical framework to the computation of the error probability of downlink mimo cellular networks by using stochastic geometry. IEEE Trans. Commun. 62(8), 2860-2879 (2014)

71. A. Rabbachin, T.Q.S. Quek, H. Shin, M.Z. Win, Cognitive network interference. IEEE J. Select. Areas Commun. 29(2), 480-493 (2011)

72. G. Samorodnitsky, M.S. Taqqu, Stable non-Gaussian Processes: Stochastic Models with Infinite Variance (Chapman \& Hall, New York, 1994)

73. C.L. Nikias, M. Shao, Signal Processing with Alpha-Stable Distributions and Applications (Wiley, New York, 1995)

74. M.Z. Win, P.C. Pinto, L.A. Shepp, A mathematical theory of network interference and its applications. Proc. IEEE 97(2), 205-230 (2009)

75. K. Gulati, B.L. Evans, J.G. Andrews, K.R. Tinsley, Statistics of co-channel interference in a field of Poisson and Poisson-Poisson clustered interferers. IEEE Trans. Signal Process. 58(12), 6207-6222 (2010)

76. R.K. Ganti, F. Baccelli, J.G. Andrews, Series expansion for interference in wireless networks. IEEE Trans. Inform. Theory 58(4), 2194-2205 (2012)

77. C.-H. Lee, M. Haenggi, Interference and outage in Poisson cognitive networks. IEEE Trans. Wireless Commun. 11(4), 1392-1401 (2012)

78. K. Gulati, B.L. Evans, S. Srikanteswara, Joint temporal statistics of interference in decentralized wireless networks. IEEE Trans. Signal Process. 60(12), 6713-6718 (2012)

79. X. Yan, L. Clavier, G.W. Peters, N. Azzaoui, F. Septier, I. Nevatn, Skew-t copula for dependence modelling of impulsive (a-stable) interference, in IEEE International Conference on Communications, ICC (2015)

80. A. Mahmood, M. Chitre, Optimal and near-optimal detection in bursty impulsive noise. IEEE J. Oceanic Eng. 42(3), 639-653 (2017)

81. A. Mahmood, M. Chitre, M.A. Armand, Baseband characterization of additive white symmetric $a$-stable noise, in Global Communications Conference (GLOBECOM), pp. 3696-3701 (2012)

82. M. Kountouris, N. Pappas, Approximating the interference distribution in large wireless networks, in 11th International Symposium on Wireless Communications Systems (ISWCS), pp. 80-84 (2014)

83. S. Weber, J.G. Andrews, N. Jindal, An overview of the transmission capacity of wireless networks. IEEE Trans. Commun. 58(12), 3593-3604 (2010)

84. E. Pitman, Subexponential distribution functions. J. Aust. Math. Soc. Ser. A 29(03), 337-347 (1980)

85. V. Chistyakov, A theorem on sums of independent positive random variables and its applications to branching random processes. Theory Prob. Appl. 9(4), 640-648 (1964)

86. P. Embrechts, C.M. Goldie, On convolution tails. Stoch. Process. Appl. 13(3), 263-278 (1982)

87. C. Klüppelberg, Subexponential distributions and integrated tails. J. Appl. Probab. 25, 132-141 (1988)

88. K.B. Athreya, P.E. Ney, Branching Processes, vol. 28 (Springer, New York, 1972)

89. S. Foss, D. Korshunov, S. Zachary, An Introduction to Heavy-Tailed and Subexponential Distributions, vol. 38 (Springer, New York, 2011)

90. K.A. Saaifan, W. Henkel, Decision boundary evaluation of optimum and suboptimum detectors in class-a interference. IEEE Trans. Commun. 61(1), 197-205 (2013)

91. T.S. Shehat, I. Marsland, M. El-Tanany: A novel framework for signal detection in alpha-stable interference, in IEEE Vehicular Technology Conference, pp. 1-5 (2010)

92. T.S. Saleh, I. Marsland, M. El-Tanany, Suboptimal detectors for alpha-stable noise: simplifying design and improving performance. IEEE Trans. Commun. 60(10), 2982-2989 (2012). https://doi.org/10.1109/TCOMM.2012.071812.10078 9

93. E.E. Kuruoglu, W.J. Fitzgerald, P.J.W. Rayner, Near optimal detection of signals in impulsive noise modeled with a symmetric $a$-stable distribution. IEEE Commun. Lett. 2, 282-284 (1998)

94. D.H. Johnson, Optimal linear detectors for additive noise channels. IEEE Trans. Signal Process. 44(12), 3079-3084 (1996) 
95. S. Niranjayan, N.C. Beaulieu, BER optimal linear combiner for signal detection in symmetric alpha-stable noise: small values of alpha. IEEE Trans. Wireless Commun. 9(3), 886-890 (2010)

96. A. Chopra, B.L. Evans, Outage probability for diversity combining in interference-limited channels. IEEE Trans. Wireless Commun. 12(2), 550-560 (2013)

97. T. Erseghe, V. Cellini, G. Dona, On UWB impulse radio receivers derived by modeling MAl as a Gaussian mixture process. IEEE Trans. Wireless Commun. 7(6), 2388-2396 (2008)

98. S. Nammi, D.K. Borah, C. Schneider, R. Thoma, Effects of impulse noise on the performance of multidimensional parity check codes, in IEEE Wireless Communications and Networking Conference, WCNC 2006, vol 4, pp. 1966-1971 (2006)

99. S. Niranjayan, N.C. Beaulieu, A myriad filter detector for UWB multiuser communication, in IEEE International Conference on Communications, ICC'08, pp. 3918-3922 (2008)

100. S. Ambike, J. Ilow, D. Hatzinakos, Detection for binary transmission in a mixture of Gaussian noise and impulsive noise modeled as an alpha-stable process. IEEE Signal Process. Lett. 1(3), 55-57 (1994)

101. H.B. Maad, A. Goupil, L. Clavier, G. Gelle, Clipping demapper for LDPC decoding in impulsive channel. IEEE Commun. Lett. 17(5), 968-971 (2013)

102. S. Zozor, J.M. Brossier, P.O. Amblard, A parametric approach to suboptimal signal detection in $a$-stable noise. IEEE Trans. Signal Processing 54(12), 4497-4509 (2006)

103. A. Spaulding, D. Middleton, Optimum reception in an impulsive interference environment-part i: coherent detection. IEEE Trans. Commun. 25(9), 910-923 (1977). https://doi.org/10.1109/TCOM.1977.1093943

104. A. Spaulding, D. Middleton, Optimum reception in an impulsive interference environment-part ii: incoherent reception. IEEE Trans. Commun. 25(9), 924-934 (1977). https://doi.org/10.1109/TCOM.1977.1093942

105. W. Gu, L. Clavier, Decoding metric study for turbo codes in very impulsive environment. IEEE Commun. Lett. 16(2), 256-258 (2012)

106. L. Seoyoung, J. Dickerson, Least Ip-norm interference suppression for DS/CDMA systems in non-Gaussian impulsive channels, in IEEE International Conference on Communications, ICC'99, vol. 2, pp. 907-911 (1999)

107. J.E. Figueroa-Lopez, S.R. Lancette, K. Lee, Y. Mi, Estimation of NIG and VG models for high frequency financial data, in Handbook for Modeling High Frequency Data in Finance, ed. by F.G. Viens, M.C. Mariani, I. Florescu (Wiley, Hoboken, 2011), pp. 1-23

108. G. Imbens, P. Johnson, R.H. Spady, Information Theoretic Approaches to Inference in Moment Condition Models (Cambridge, National Bureau of Economic Research, 1995)

109. M.K. Varanasi, B. Aazhang, Parametric generalized Gaussian density estimation. J. Acoust. Soc. Am. 86, 1404 (1989)

110. M.N. Do, M. Vetterli, Wavelet-based texture retrieval using generalized Gaussian density and Kullback-Leibler distance. IEEE Trans. Image Process. 11(2), 146-158 (2002). https://doi.org/10.1109/83.982822

111. Y. Mestrah, A. Savard, A. Goupil, G. Gelle, L. Clavier, Robust and simple log-likelihood approximation for receiver design, in 2019 IEEE Wireless Communications and Networking Conference (WCNC), pp. 1-6 (2019). https://doi. org/10.1109/WCNC.2019.8886114

112. C. Boyd, R. Kotaba, O. Tirkkonen, P. Popovski, Non-orthogonal contention-based access for URLLC devices with frequency diversity, in 2019 IEEE 20th International Workshop on Signal Processing Advances in Wireless Communications (SPAWC), pp. 1-5 (2019). https://doi.org/10.1109/SPAWC.2019.8815506

113. G.R. Hossack, G.W. Peters, K. Hayes, Estimating nonlinear ecological state space models with flexible observation error. Methods Ecol. Evolut. 3(6), 1028-1038 (2012)

114. O.E. Barndorff-Nielsen, N. Shephard, Non-Gaussian Ornstein-Uhlenbeck-based models and some of their uses in financial economics. J. R. Stat. Soc. Ser. B Stat. Methodol. 63(2), 167-241 (2001)

115. T.C. Aysal, K.E. Barner, Generalized mean-median filtering for robust frequency-selective applications. IEEE Trans. Signal Process. 55(3), 937-948 (2007). https://doi.org/10.1109/TSP.2006.888882

116. D.S. Pham, Y.H. Leung, A. Zoubir, R. Brcic, On the computational aspect of robust multiuser detection, in Proceedings of the 3rd IEEE International Symposium on Signal Processing and Information Technology, 2003. ISSPIT 2003, pp. 22-25 (2003)

117. H. Hamad, G.M. Kraidy, Performance analysis of convolutional codes over the Bernoulli-Gaussian impulsive noise channel, in 15th Canadian Workshop on Information Theory (CWIT), Quebec City, QC, Canada (2017)

118. J.V.M. Cardoso, W.J.L. Queiroz, H. Liu, M.S. Alencar, On the performance of the energy detector subject to impulsive noise in $\kappa-\mu, a-\mu$, and $\eta-\mu$ fading channels. Tsinghua Sci. Technol. 22(4), 360-367 (2017)

119. M. Haenggi, R.K. Ganti, Interference in large wireless networks. Found. Trends Netw. 3(2), 127-248 (2009)

\section{Publisher's Note}

Springer Nature remains neutral with regard to jurisdictional claims in published maps and institutional affiliations. 